\title{
FINITE ELEMENT FORMULATIONS FOR LARGE DEFORMATION DYNAMIC ANALYSIS
}

\author{
KI_AUS-JÜRGEN BATHE* \\ Civil Engineering Department, University of California, Berkeley, California, U.S.A. \\ EKKEHARD RAMM† \\ University of Stutgart, West Germany \\ EDWARD L. WILSON $\ddagger$ \\ Civil Engineering Department, University of California, Berkeley, California, U.S.A.
}

\begin{abstract}
SUMMARY
Starting from continuum mechanics principles, finite element incremental formulations for non-linear static and dynamic analysis are reviewed and derived. The aim in this paper is a consistent summary, comparison, and evaluation of the formulations which have been implemented in the search for the most effective procedure. The general formulations include large displacements, large strains and material non-linearities. For specific static and dynamic analyses in this paper, elastic, hyperelastic (rubber-like) and hypoelastic elastic-plastic materials are considered. The numerical solution of the continuum mechanics equations is achieved using isoparametric finite element discretization. The specific matrices which need be calculated in the formulations are presented and discussed. To demonstrate the applicability and the important differences in the formulations, the solution of static and dynamic problems involving large displacements and large strains are presented.
\end{abstract}

\section{INTRODUCTION}

In non-linear dynamic finite element analysis involving large displacements, large strains and material non-linearities, it is necessary to resort to an incremental formulation of the equations of motion. Various formulations are used in practice (see References). Some procedures are general and others are restricted to account for material non-linearities only, or for large displacements but not for large strains, or the formulation may only be applicable to certain types of elements. Limited results have been obtained in dynamic non-linear analysis involving large displacements and large strains.

Currently, the general purpose non-linear finite element analysis program NONSAP is being developed at the University of California, Berkeley. ${ }^{2}$ An important aspect in the development of the program is to assess which general finite element formulation should be implemented.

In dynamic analysis numerical time integration of the finite element equations of motion is required. Extensive research is currently being devoted towards the development of stable and accurate integration schemes. ${ }^{1.5 .20 .31}$ However, it need be realized that a proper evaluation and use of an integration method is only possible if a consistent non-linear finite element formulation is used.

* Assistant Research Engineer.

$\dagger$ Research Fellow of Deutsche Forschungsgemeinschaft. Formerly Visiting Scholar, University of California.

$\ddagger$ Professor.

Received 18 February 1974

() 1975 by John Wiley \& Sons, Ltd 
The earliest non-linear finite element analyses were essentially based on extensions of linear analyses and have been developed for specific applications (for a comprehensive list of References, see the books by Oden ${ }^{33}$ and Zienkiewicz ${ }^{43}$ ). The procedures were primarily developed on an intuitive basis in order to obtain solutions to the specific problems considered. However, to provide general analysis capabilities using isoparametric (and related) elements a general formulation need be used. The isoparametric finite element discretization procedure has proved to be very effective in many applications, and lately it has been shown that general non-linear formulations based on principles of continuum mechanics can be efficiently implemented.

Basically, two different approaches have been pursued in incremental non-linear finite element analysis. In the first, static and kinematic variables are referred to an updated configuration in each load step. This procedure is generally called Eulerian, moving co-ordinate or updated formulation. Murray and Wilson, ${ }^{28}$ Felippa, ${ }^{9}$ Yaghmai, ${ }^{39}$ Yaghmai and Popov, ${ }^{40}$ Farhoomand, ${ }^{8}$ Sharifi and Popov, ${ }^{35}$ Yamada, ${ }^{41}$ Stricklin and many others, ${ }^{38}$ Heifitz and Costantino, ${ }^{15}$ Belytschko and $\mathrm{Hsieh}^{6}$ have presented some form of this formulation.

In the second approach, which is generally called Lagrangian formulation, all static and kinematic variables are referred to the initial configuration. This procedure is used by Oden, ${ }^{32.33}$ Marcal, ${ }^{26}$ Hibbitt et al, ${ }^{16}$ Larsen, ${ }^{22}$ McNamara, ${ }^{30}$ Sharifi and Yates, ${ }^{36}$ Stricklin and many others, ${ }^{37,38}$ Haug and Powell. ${ }^{13}$ A survey paper of the Lagrangian formulation in static analysis was presented by Hibbitt et al, ${ }^{16}$ where it is stated that additional research is required for use of an equivalently consistent updated formulation.

It is apparent that with the different formulations available, in the development of a general purpose non-linear dynamic analysis program a decision need be made on the procedure to be used. An important consideration is that using any formulation based on continuum mechanics principles, in which all non-linear effects are included, the same results should be obtained in the analyses. Stricklin and many others, discussed a moving co-ordinate formulation and a Lagrangian formulation and pointed out that the latter is more general and computationally more efficient. ${ }^{38}$ Yamada compared an Eulerian and Lagrangian formulation and predicted for a simple truss structure a maximum difference of about 25 per cent in the displacements. ${ }^{41}$ Dupuis and many others, analyzed arches using the Lagrangian and an updated formulation and also calculated a much different response by either formulation. ${ }^{7}$

The purpose of this paper is to present and compare in detail the general formulations that have been implemented in program NONSAP, and to show their general applicability in nonlinear static and dynamic analysis. The formulations are termed total Lagrangian and updated Lagrangian formulations and they are based on the work of the authors cited above. For specific solutions in this paper, elastic, hyperelastic, and hypoelastic materials are considered.

The procedures are derived from the basic principle of virtual work and are valid for non-linear material behaviour, large displacements and large strains. It is pointed out that, in theory, there is no difference in the formulations. Any differences in the numerical results arise from the fact that different descriptions of material behaviour are assumed, and if the material constants are transformed appropriately, identical numerical results are obtained. Therefore, the question of which formulation should be used merely depends on the relative numerical effectiveness of the methods. In the paper specific attention is directed to the numerical efficiency of either formulation.

To demonstrate the applicability and the important differences in the formulations, the numerical operations required for solution are studied and a variety of sample solutions are presented. These include the large displacement static and dynamic analysis of a cantilever, the large displacement and large strain static and dynamic analysis of a rubber-like material and the static and dynamic, elastic and elastic-plastic large displacement analysis of arches and shells. 


\section{FORMULATION OF THE CONTINUUM MECHANICS INCREMENTAL EQUATIONS OF MOTION}

Consider the motion of a body in a Cartesian co-ordinate system as shown in Figure 1. The aim is to evaluate the equilibrium positions of the body at the discrete time points $0, \Delta t, 2 \Delta t$, $3 \Delta t, \ldots$, where $\Delta t$ is an increment in time. Assume that the solution for the kinematic and static variables for all time steps from time 0 to time $t$, inclusive, have been solved, and that the solution for time $t+\Delta t$ is required next. It is noted that the solution process for the next required equilibrium position is typical and would be applied repetitively until the complete solution path has been solved.

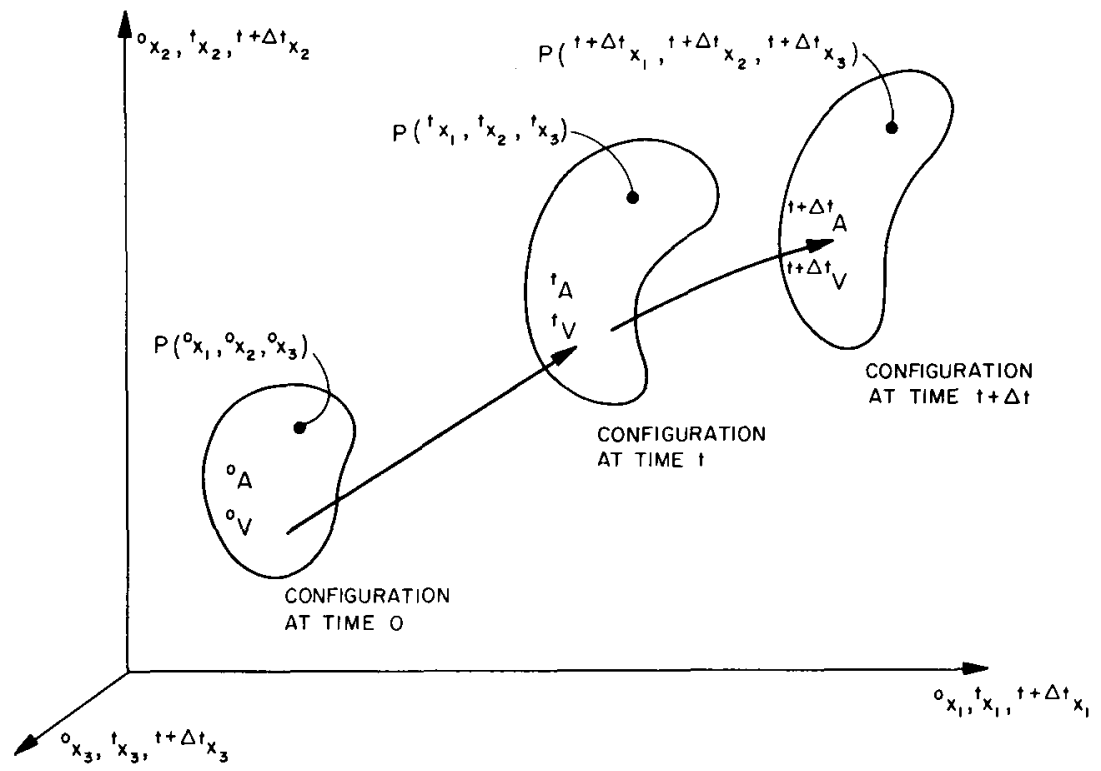

Figure 1. Motion of body in Cartesian co-ordinate system

\section{Nomenclature}

It is useful at this point to lay out the notation which will be employed.

The motion of the body is considered in a fixed Cartesian co-ordinate system, Figure 1, in which all kinematic and static variables are defined.

The co-ordinates describing the configuration of the body at time 0 are ${ }^{0} x_{1},{ }^{0} x_{2},{ }^{0} x_{3}$, at time $t$ are ${ }^{t} x_{1},{ }^{t} x_{2},{ }^{t} x_{3}$, and at time $t+\Delta t$ are ${ }^{t+\Delta t} x_{1},{ }^{t+\Delta t} x_{2},{ }^{t+\Delta t} x_{3}$, where the left superscripts refer to the configuration of the body and the subscripts to the co-ordinate axes.

The notation for the displacements of the body is similar to the notation for the co-ordinates; at time $t$ the displacements are ${ }^{t} u_{i}, i=1,2,3$ and at time $t+\Delta t$ the displacements are ${ }^{t+\Delta t} u_{i}$, $i=1,2,3 ;$ therefore

$$
\left.\begin{array}{rl}
{ }^{t} x_{i} & ={ }^{0} x_{i}+{ }^{t} u_{i} \\
{ }^{t+\Delta t} x_{i} & ={ }^{0} x_{i}+{ }^{t+\Delta t} u_{i}
\end{array}\right\} \quad i=1,2,3
$$

The unknown increments in the displacements from time $t$ to $t+\Delta t$ are denoted as

$$
u_{i}={ }^{t+\Delta t} u_{i}-{ }^{t} u_{i} ; \quad i=1,2,3
$$


During motion of the body, its volume, surface area, mass density, stresses and strains are changing continuously. The specific mass, area and volume of the body at times $0, t$ and $t+\Delta t$ are denoted by ${ }^{0} \rho,{ }^{t} \rho,{ }^{t+\Delta t} \rho:{ }^{0} A,{ }^{t} A,{ }^{t+\Delta t} A$ : and ${ }^{0} V,{ }^{t} V,{ }^{t+\Delta t} V:$ respectively.

Since the configuration of the body at time $t+\Delta t$ is not known, the applied forces, stresses and strains are referred to a known equilibrium configuration. In analogy to the notation used for co-ordinates and displacements a left superscript indicates in which configuration the quantity (body force, surface traction, stress, ... ) occurs; in addition, a left subscript indicates with respect to which configuration the quantity is measured.

The surface and body force components per unit mass at time $t+\Delta t$, but measured in configuration $t$, are ${ }^{t+\Delta t} t_{k},{ }^{t+\Delta t} f_{k}, k=1,2,3$.

Considering stresses, the Cartesian components of the Cauchy stress tensor at time $t+\Delta t$ are denoted by ${ }^{i+\Delta t} \tau_{i j}$ (since Cauchy stresses are always referred to the configuration in which they do occur ${ }^{t+\Delta t} \tau_{i j} \equiv{ }_{i+\Delta t}^{t+\Delta t} \tau_{i j}$ ), and the Cartesian components of the 2nd Piola-Kirchhoff stress tensor corresponding to the configuration at time $t+\Delta t$ but measured in configuration at time $t$ are denoted by ${ }^{t+\Delta t} S_{i j}$.

Considering strains, the Cartesian components of Cauchy's infinitesimal strain tensor referred to the configuration at time $t+\Delta t$ are denoted by ${ }_{t+\Delta t} e_{i j}$; and the Cartesian components of the Green-Lagrange strain tensor using the displacements from the configuration at time $t$ to the configuration at time $t+\Delta t$, and referred to the configuration at time $t$ are denoted by ${ }^{t+\Delta t}{ }_{t} \varepsilon_{i j}$.

The reference configurations, which will be used for applied forces, Kirchhoff-Piola stresses and Green-Lagrange strains, are those at time 0 and at time $t$.

In the formulation of the governing equilibrium equations derivatives of displacements and co-ordinates need be considered. In the notation adopted, a comma denotes differentiation with respect to the co-ordinate following, and the left time subscripts indicate the configuration in which this co-ordinate is measured; thus, for example,

$$
{ }_{0}^{t+\Delta t} u_{i, j}=\frac{\partial^{t+\Delta t} u_{i}}{\partial^{0} x_{j}} \quad \text { and } \quad{ }_{t+\Delta t}^{0} x_{m, n}=\frac{\partial^{0} x_{m}}{\partial^{t+\Delta t} x_{n}}
$$

\section{Principle of virtual displacements}

With the notation having been explained briefly, consider again the body in Figure 1. Since the solution is known at all discrete time points $0, \Delta t, 2 \Delta t, \ldots, t$, the basic aim of the formulation is to establish an equation of virtual work from which the unknown static and kinematic variables in the configuration at time $t+\Delta t$ can be solved. Since the isoparametric displacement based finite element procedure shall be employed for numerical solution, the principle of virtual displacements is used to express the equilibrium of the body in the configuration at time $t+\Delta t$. The principle of virtual displacements requires that

$$
\int_{t+\Delta t V}{ }^{t+\Delta t} \tau_{i j} \delta_{t+\Delta t} e_{i j}{ }^{t+\Delta t} \mathrm{~d} v={ }^{t+\Delta t} \mathscr{R}
$$

where ${ }^{t+\Delta t} \mathscr{R}$ is the external virtual work expression,

$$
{ }^{t+\Delta t} \mathscr{R}=\int_{t+\Delta t A}{ }^{t+\Delta t} t_{k} t_{k} \delta u_{k}{ }^{t+\Delta t} \mathrm{~d} a+\int_{t+\Delta t V}{ }^{t+\Delta t} \rho_{t+\Delta t}^{t+\Delta t} f_{k} \delta u_{k}{ }^{t+\Delta t} \mathrm{~d} v
$$

In equations (1) and (2) $\delta u_{k}$ is a (virtual) variation in the current displacement components ${ }^{t+\Delta t} u_{k}$, and $\delta_{t+\Delta t} e_{i j}$ are the corresponding (virtual) variations in strains, i.e.

$$
\delta_{t+\Delta t} e_{i j}=\delta \frac{1}{2}\left(t+\Delta t u_{i, j}+{ }_{t+\Delta t} u_{j, i}\right)
$$


It need be noted that in equation (1) and the equations to follow the summation convention of tensor notation is implied.

Equation (1) cannot be solved directly since the configuration at time $t+\Delta t$ is unknown. A solution can be obtained by referring all variables to a known previously calculated equilibrium configuration. For this purpose, in principle, any one of the already calculated equilibrium configurations could be used. In practice, however, the choice lies essentially bet ween two different formulations, namely, the total Lagrangian formulation (T.L.) and the updated Lagrangian (U.L.) formulation, which are presented in the following sections.

\section{Total Lagrangian formulation}

The formulation called here total Lagrangian (T.L.) formulation is generally referred to as Lagrangian formulation and has been used a great deal in static analysis. ${ }^{14,16,26.38}$

In the formulation all variables in equations (1) and (2) are referred to the initial configuration at time 0 of the body. The applied forces in equation (2) are evaluated using

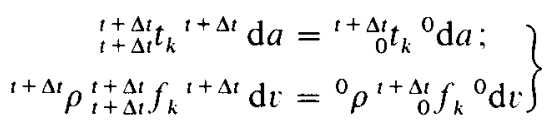

where it is assumed that the direction and magnitude of the forces ${ }^{t+\Delta_{0} t} t_{k}$ and ${ }^{0} \rho^{t+\Delta_{t}} f_{k}$ are independent of the specific configuration at time $t+\Delta t$. Loading conditions that depend on the deformations will be considered later.

The volume integral of Cauchy stresses times variations in infinitesimal strains in equation (1) can be transformed to give ${ }^{25}$

$$
\int_{t+\Delta t \boldsymbol{V}}{ }^{1+\Delta t} \tau_{i j} \delta_{t+\Delta t} e_{i j}{ }^{t+\Delta t} \mathrm{~d} v=\int_{{ }^{V} V}{ }^{t+\Delta t}{ }_{0} S_{i j} \delta^{t+\Delta t}{ }_{0} \varepsilon_{i j}{ }^{o} \mathrm{~d} t
$$

where ${ }^{t+\Delta t}{ }_{0} S_{i j}=$ Cartesian components of the 2nd Piola-Kirchhoff stress tensor corresponding to the configuration at time $t+\Delta t$ but measured in the configuration at time 0 ,

$$
{ }^{t+\Delta t} S_{i j}=\frac{{ }^{0} \rho}{t+\Delta t}{ }^{t+\Delta t}{ }^{0} x_{i, s}{ }^{t+\Delta t} \tau_{s r t+\Delta t} x_{j, r}
$$

and $\delta^{t+\Delta t}{ }_{0} \varepsilon_{i j}=$ variations in the Cartesian components of the Green-Lagrange strain tensor in the configuration at time $t+\Delta t$, referred to the configuration at time 0 ,

$$
\delta^{t+\Delta t} \varepsilon_{i j}=\delta \frac{1}{2}\left({ }^{t+\Delta t} u_{0} u_{i, j}+{ }^{t+\Delta t} u_{j . i}+{ }_{0}^{t+\Delta t} u_{k, i}{ }^{t+\Delta t}{ }_{0} u_{k, j}\right)
$$

It should be noted that the integral of Piola-Kirchhoff stresses times variations in the GreenLagrange strains is defined over the initial configuration at time 0 of the body.

Substituting the relations in equations (3) and (4) into equations (1) and (2), the following equilibrium equation for the body in the configuration at time $t+\Delta t$ but referred to the configuration at time 0 is obtained,

$$
\int_{{ }_{0} V}{ }^{t+\Delta t}{ }_{0} S_{i j} \delta^{t+\Delta t} \varepsilon_{i j}{ }^{0} \mathrm{~d} v={ }^{t+\Delta t} \mathscr{R}
$$


where $^{t+\Delta t} \mathscr{R}$ is now calculated using

$$
{ }^{t+\Delta t} \mathscr{R}=\int_{0_{A}}{ }^{t+\Delta t}{ }_{0} t_{k} \delta u_{k}{ }^{0} \mathrm{~d} a+\int_{{ }^{V} V}{ }^{0} \rho^{t+\Delta t}{ }_{0} f_{k} \delta u_{k}{ }^{0} \mathrm{~d} v
$$

Since the stresses ${ }^{t+\Delta t} S_{i j}$ and strains ${ }^{t+\Delta t} \varepsilon_{i j}$ are unknown, for solution, the following incremental decompositions are used

$$
\begin{aligned}
{ }^{t+}{ }_{0}^{\Delta t} S_{i j} & ={ }_{0}^{t} S_{i j}+{ }_{0} S_{i j} \\
{ }^{t+\Delta t} \varepsilon_{i j} & ={ }_{0}^{t} \varepsilon_{i j}+{ }_{0} \varepsilon_{i j}
\end{aligned}
$$

where ${ }_{0}^{t} S_{i j}$ and ${ }_{0}^{t} \varepsilon_{i j}$ are the known 2nd Piola-Kirchhoff stresses and Green-Lagrange strains in the configuration at time $t$. Using the displacement definition of the Green-Lagrange strain tensor, it follows from equation (10) that $\delta^{t+{ }_{0}^{t}} \varepsilon_{i j}=\delta_{0} \varepsilon_{i j}$ and

$$
{ }_{0} \varepsilon_{i j}={ }_{0} e_{i j}+{ }_{0} \eta_{i j}
$$

where

$$
\begin{gathered}
{ }_{0} e_{i j}=\frac{1}{2}\left({ }_{0} u_{i, j}+{ }_{0} u_{j, i}+{ }_{0}^{t} u_{k, i} 0 u_{k, j}+{ }_{0} u_{k, i}{ }_{0} u_{k, j}\right) \\
{ }_{0} \eta_{i j}=\frac{1}{2}{ }_{0} u_{k, i} u_{k, j}
\end{gathered}
$$

The incremental 2nd Piola-Kirchhoff stresses ${ }_{0} S_{i j}$ are related to the incremental GreenLagrange strains ${ }_{0} \varepsilon_{i j}$ using the constitutive tensor ${ }_{0} C_{i j r s}$, i.e.

$$
{ }_{0} S_{i j}={ }_{0} C_{i j r s 0} \varepsilon_{r s}
$$

Equation (7) can now be written as

$$
\int_{{ }^{V} V}{ }_{0} C_{i j r s} 0^{\varepsilon_{r s}} \delta_{0} \varepsilon_{i j}{ }^{0} \mathrm{~d} v+\int_{{ }^{V} V}{ }_{0} S_{i j} \delta_{0} \eta_{i j}{ }^{0} \mathrm{~d} v={ }^{t+\Delta t} \mathscr{R}-\int_{{ }_{0} V}{ }_{0} S_{i j} \delta_{0} e_{i j}{ }^{0} \mathrm{~d} v
$$

which represents a non-linear equation for the incremental displacements $u_{i}$.

\section{Updated Lagrangian formulation}

Most updated formulations previously used are approximate in that they are restricted to small strains or even constant strain conditions within each finite element used for numerical solution. ${ }^{6.28}$ However, Yaghmai introduced a general procedure, and the U.L. formulation given here is largely based on his work. ${ }^{39}$

In the U.L. formulation all variables in equations (1) and (2) are referred to the configuration at time $t$, i.e. the updated configuration of the body. By an analogous procedure to the derivation of the T.L. formulation, equation (1) is in this case transformed to

$$
\int_{{ }^{t} V}{ }^{t+\Delta t} S_{i j} \delta^{t+\Delta t} \varepsilon_{i j}{ }^{t} \mathrm{~d} v={ }^{t+\Delta t} \mathscr{R}
$$

where ${ }^{t+\Delta t} S_{i j}=$ Cartesian components of the 2nd Piola-Kirchhoff stress tensor and ${ }^{t+\Delta t}{ }_{t} \varepsilon_{i j}=$ Cartesian components of the Green-Lagrange strain tensor from the configuration at time $t$ to the configuration at time $t+\Delta t$ and referred to the configuration at time $t$. The quantities ${ }^{t+\Delta t} S_{i j}$ and ${ }^{t+\Delta t} \varepsilon_{i j}$ are defined by equations (5) and (6), respectively, if the superscript and subscript ' 0 ' is replaced by ' $t$ ' and displacements are measured from the configuration at time $t$. Since deformation independent loading is considered ${ }^{t+\Delta t} \mathscr{R}$ is evaluated as in the T.L. formulation. 
The incremental stress decomposition used in this case is

$$
{ }^{t+\Delta t} S_{i j}={ }^{t} \tau_{i j}+{ }_{t} S_{i j}
$$

where ${ }^{t} \tau_{i j}=$ Cartesian components of the Cauchy stress tensor and ${ }_{t} S_{i j}=$ Cartesian components of the 2nd Piola-Kirchhoff stress increment tensor referred to the configuration at time $t$. Considering the strain increments ${ }^{t+\Delta t} \varepsilon_{i j}$, the following $\cdot$ relations hold

$$
\begin{gathered}
{ }^{t+\Delta t} \varepsilon_{i j} \varepsilon_{i j}={ }_{t} \varepsilon_{i j} \\
{ }_{t} \varepsilon_{i j}={ }_{t} e_{i j}+{ }_{t} \eta_{i j}
\end{gathered}
$$

where

$$
\begin{aligned}
& { }_{t} e_{i j}=\frac{1}{2}\left({ }_{t} u_{i, j}+{ }_{\imath} u_{j, i}\right) \\
& { }_{t} \eta_{i j}=\frac{1}{2}{ }_{\imath} u_{k, i}, u_{k, j}
\end{aligned}
$$

The constitutive relation between stress and strain increments used now is

$$
{ }_{i} S_{i j}={ }_{t} C_{i j r s}{ }_{i} \varepsilon_{r s}
$$

and equation (16) can be rewritten as

$$
\int_{t V}{ }_{t} C_{i j r s t} \varepsilon_{r s} \delta_{t} \varepsilon_{i j}{ }^{t} \mathrm{~d} v+\int_{{ }^{t} V}{ }^{t} \tau_{i j} \delta_{t} \eta_{i j}{ }^{t} \mathrm{~d} v={ }^{t+\Delta t} \mathscr{R}-\int_{{ }^{t} V}{ }^{t} \tau_{i j} \delta_{t} e_{i j}{ }^{t} \mathrm{~d} v
$$

which, as equation (15), is a non-linear equation in the incremental displacements $u_{i}$.

\section{Linearization of equilibrium equations}

It should be noted that equations (15) and (23) are, theoretically, equivalent and provided the appropriate constitutive relations are used, the equations yield identical solutions. However, as will be seen, the finite element matrices established for solution are different.

The solution of equation (15) and of equation (23) cannot be calculated directly, since they are nonlinear in the displacement increments. Approximate solutions can be obtained by assuming that in equation (15) ${ }_{0} \varepsilon_{i j}={ }_{0} e_{i j}$ and in equation (23) ${ }_{t} \varepsilon_{i j}={ }_{t} e_{i j}$. This means that, in addition to using $\delta_{0} \varepsilon_{i j}=\delta_{0} e_{i j}$ and $\delta_{t} \varepsilon_{i j}=\delta_{t} e_{i j}$, respectively, the incremental constitutive relations employed are

and

$$
{ }_{0} S_{i j}={ }_{0} C_{i j r s} e_{r s}
$$

$$
{ }_{t} S_{i j}={ }_{t} C_{i j r s t} e_{r s}
$$

\section{FINITE ELEMENT SOLUTION}

In the T.L. formulation the approximate equilibrium equation to be solved is

$$
\int_{0_{V}}{ }_{0} C_{i j r s} e_{0} e_{r s} \delta_{0} e_{i j}{ }^{0} \mathrm{~d} v+\int_{0_{V}}{ }_{0}^{t} S_{i j} \delta_{0} \eta_{i j}{ }^{0} \mathrm{~d} v={ }^{t+\Delta t} \mathscr{R}-\int_{0_{V}}{ }_{0}^{t} S_{i j} \delta_{0} e_{i j}{ }^{0} \mathrm{~d} v
$$

whereas in the U.L. formulation the equation is

$$
\int_{t V}{ }_{t} C_{i j r s t} e_{r s} \delta_{t} e_{i j}{ }^{t} \mathrm{~d} v+\int_{{ }^{V} V}{ }^{t} \tau_{i j} \delta_{t} \eta_{i j}{ }^{t} \mathrm{~d} v={ }^{t+\Delta t} \mathscr{R}-\int_{t V}{ }^{t} \tau_{i j} \delta_{t} e_{i j}{ }^{t} \mathrm{~d} v
$$


Equations (26) and (27) are linear equations in the incremental displacements and are used as the basis for isoparametric finite element analysis. ${ }^{32,43}$ Referring to the standard procedures for assembling the structure matrices, attention need only be given to the derivation of the matrices corresponding to a single element.

\section{Finite element matrices}

In the isoparametric element solution the co-ordinates and displacements are interpolated using

$$
\left.\begin{array}{cc}
{ }^{0} x_{i}=\sum_{k=1}^{N} h_{k}{ }^{0} x_{i}^{k} ; \quad{ }^{t} x_{i}=\sum_{k=1}^{N} h_{k}{ }^{t} x_{i}^{k} \\
{ }^{t+\Delta t} x_{i}=\sum_{k=1}^{N} h_{k}{ }^{t+\Delta t} x_{i}^{k}
\end{array}\right\} \quad r=1,2,3
$$

where ${ }^{t} x_{i}^{k}$ is the co-ordinate of nodal point $k$ corresponding to direction $i$ at time $t,{ }^{\prime} u_{i}^{k}$ is defined similarly to ${ }^{t} x_{i}^{k}, h_{k}$ is the interpolation function corresponding to nodal point $k$, and $N$ is the number of element nodal points. ${ }^{43}$

Using equations (28) and (29) to evaluate the displacement derivatives required in the integrals, equation (26) becomes, considering a single element

$$
\left({ }_{0}^{t} \mathbf{K}_{L}+{ }_{0}^{t} \mathbf{K}_{N L}\right) \mathbf{u}={ }^{t+\Delta t} \mathbf{R}-{ }_{0}^{t} \mathbf{F}
$$

where ${ }_{0}^{t} K_{L} \mathbf{u},{ }_{0}^{t} K_{N L} \mathbf{u}$ and ${ }_{0}^{t} \mathbf{F}$ are obtained from the finite element evaluation of $\int_{{ }_{0}}{ }_{0} C_{i j r s} e_{r s} \times$ $\delta_{0} e_{i j}{ }^{0} \mathrm{~d} v, \int_{O_{V}}{ }_{0}^{t} S_{i j} \delta_{0} \eta_{i j}{ }^{0} \mathrm{~d} v$, and $\int_{O_{V}}{ }_{0}^{t} S_{i j} \delta_{0} e_{i, j}{ }^{0} \mathrm{~d} v$, respectively, i.e.

$$
\begin{gathered}
{ }_{0}^{t} \mathbf{K}_{L}=\int_{{ }_{0} V}{ }_{0}^{t} \mathbf{B}_{L}^{\mathrm{T}}{ }_{0} \mathbf{C}_{0}^{t} \mathbf{B}_{L}{ }^{0} \mathrm{~d} v \\
{ }_{0}^{t} \mathbf{K}_{N L}=\int_{{ }_{0} V}{ }_{0}^{t} \mathbf{B}_{N L}^{\mathrm{T}}{ }_{0}^{t} \mathbf{S}_{0}^{t} \mathbf{B}_{N L}{ }^{o} \mathrm{~d} v \\
{ }_{0}^{t} \mathbf{F}=\int_{{ }_{0} V}{ }_{0}^{t} \mathbf{B}_{L}^{\mathrm{T}}{ }_{0}^{t} \hat{\mathbf{S}}^{0} \mathrm{~d} v
\end{gathered}
$$

The vector ${ }^{t+\Delta t} \mathbf{R}$ in equation (30) is obtained from the finite element evaluation of equation (8) in the usual way. ${ }^{43}$ In the above equations, ${ }_{0}^{t} \mathbf{B}_{L}$ and ${ }_{0}^{t} \mathbf{B}_{N L}$ are linear and non-linear straindisplacement transformation matrices, ${ }_{0} \mathbf{C}$ is the incremental material property matrix, ${ }_{0}^{t} \mathbf{S}$ is a matrix of 2nd Piola-Kirchhoff stresses, and ${ }_{0}^{i} \hat{\mathbf{S}}$ is a vector of these stresses. All matrix elements correspond to the configuration at time $t$ and are defined with respect to the configuration at time 0 .

Similarly, the finite element solution of equation (27), which was obtained using the U.L. formulation, results into

$$
\left({ }_{i}^{t} \mathbf{K}_{L}+{ }_{t}^{t} \mathbf{K}_{N L}\right) \mathbf{u}={ }^{i+\Delta t} \mathbf{R}-{ }_{t}^{t} \mathbf{F}
$$

where

$$
{ }_{i}^{t} \mathbf{K}_{L}=\int_{{ }^{t} V}{ }_{t}^{t} \mathbf{B}_{L}^{\mathrm{T}}{ }_{t} \mathbf{C}_{t}^{t} \mathbf{B}_{L}{ }^{t} \mathrm{~d} v
$$




$$
{ }_{i}^{t} \mathbf{K}_{N L}=\int_{{ }^{\prime} V}{ }_{t}^{t} \mathbf{B}_{N L}^{\mathrm{T}}{ }^{t} \tau{ }_{t}^{t} \mathbf{B}_{N L}{ }^{t} \mathrm{~d} v
$$

and

$$
{ }_{t}^{t} \mathbf{F}=\int_{{ }_{t}}{ }_{t}^{t} \mathbf{B}_{L}^{\mathrm{T}}{ }^{t} \hat{\mathrm{\tau}}^{t} \mathrm{~d} v
$$

In Equations (35) to (37) the elements of the linear and non-linear strain-displacement transformation matrices ${ }_{t}^{t} \mathbf{B}_{L}$ and ${ }_{i}^{t} \mathbf{B}_{N L}$, respectively, and the elements of the incremental material property matrix ${ }_{t} \mathbf{C}$ correspond to and are defined with respect to the configuration at time $t$, ${ }^{i} \tau$ is a matrix and ${ }^{t} \hat{\boldsymbol{\tau}}$ is a vector of Cauchy stresses in the configuration at time $t$.

It should be noted that the elements of the matrices in equations (30) to (37) are functions of the natural element co-ordinates and that the volume integrations are performed using a coordinate change from Cartesian to natural co-ordinates. ${ }^{43}$ Table I gives the strain-displacement and stress matrices used for two-dimensional (plane stress, plane strain and axisymmetric) analysis in the U.L. and T.L. formulations. Figure 2 shows the 4 to 8 variable-number-nodes element that has been used in the sample solutions. ${ }^{3}$
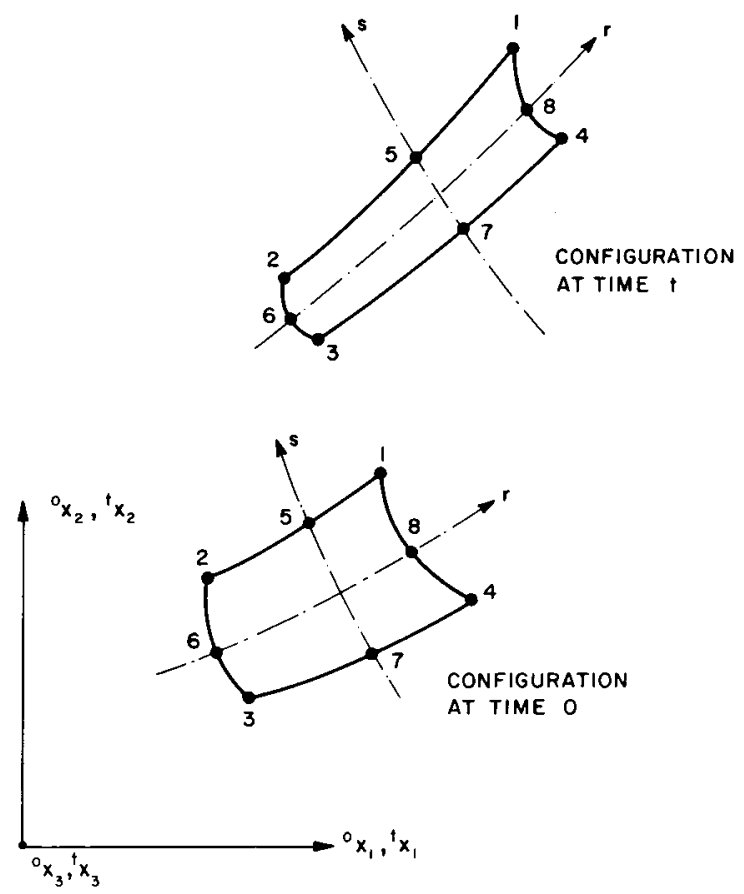

Figure 2. Two-dimensional element shown in the global ${ }^{t} x_{1}-{ }^{t} x_{2}$ plane

Dynamic analysis

In dynamic analysis, the applied body forces include inertia forces. Assuming that the mass of the body considered is preserved, the mass matrix can in both formulations be evaluated prior to the time integration using the initial configuration at time 0 as reference. Employing the standard finite element formulation to evaluate the element mass matrix, ${ }^{43}$ the incremental equilibrium equation for a single element in the T.L. formulation is

$$
\left({ }_{0}^{t} \mathbf{K}_{L}+{ }_{0}^{t} \mathbf{K}_{N L}\right) \mathbf{u}={ }^{t+\Delta t} \mathbf{R}-{ }_{0}^{t} \mathbf{F}-\mathbf{M}^{t+\Delta t_{i \mathbf{i}}}
$$




\section{Total Lagrangian formulation}

\section{Incremental strains}

$\left.{ }_{0} \varepsilon_{11}={ }_{0} u_{1,1}+{ }_{0}^{t} u_{1,1} u_{1,1}+{ }_{0} u_{2,1} u_{0,1}+\frac{1}{2}\left[\left({ }_{0} u_{1,1}\right)^{2}+{ }_{0} u_{2,1}\right)^{2}\right]$

$\left.{ }_{0} \varepsilon_{22}={ }_{0} u_{2,2}+{ }_{0} u_{1,2}{ }_{0} u_{1,2}+{ }_{0}{ }_{0} u_{2,2}{ }_{0} u_{2,2}+\frac{1}{2}\left[\left({ }_{0} u_{1,2}\right)^{2}+{ }_{0} u_{2,2}\right)^{2}\right]$

${ }_{0} \varepsilon_{12}=\frac{1}{2}\left[{ }_{0} u_{1,2}+{ }_{0} u_{2,1}\right]+\frac{1}{2}\left[{ }_{0}^{t} u_{1,1} u_{1,2}+{ }_{0}^{t} u_{2,1}{ }_{0} u_{2,2}+{ }_{0}^{t} u_{1,2} u_{1,1}+{ }_{0}^{t} u_{2,2}{ }_{0} u_{2,1}\right]+\frac{1}{2}\left[{ }_{0} u_{1,10} u_{1,2}+{ }_{0} u_{2,1} u_{0,2}\right]$

${ }_{0} \varepsilon_{33}=\frac{u_{1}}{{ }^{0} x_{1}}+\frac{{ }^{t} u_{1} u_{1}}{\left({ }^{0} x_{1}\right)^{2}}+\frac{1}{2}\left(\frac{u_{1}}{{ }^{0} x_{1}}\right)^{2} \quad$ (axisymmetric analysis)

where

$$
{ }_{0} u_{i, j}=\frac{\partial u_{i}}{\partial^{0} x_{j}} ; \quad{ }_{0}^{t} u_{i, j}=\frac{\partial^{t} u_{i}}{\partial^{0} x_{j}}
$$

\section{Linear strain-displacement transformation matrix}

\section{Using}

$$
{ }_{0} \mathbf{e}={ }_{0}^{t} \mathbf{B}_{L} \mathbf{u}
$$

where

$$
{ }_{0} \mathbf{e}^{\mathrm{T}}=\left[\begin{array}{llll}
{ }_{0} e_{11} e e_{22} & 2{ }_{0} e_{12}{ }_{0} e_{33}
\end{array}\right] ; \quad \mathbf{u}^{\mathrm{T}}=\left[u_{1}^{1} u_{2}^{1} u_{1}^{2} u_{2}^{2} \ldots u_{1}^{N} u_{2}^{N}\right]
$$

and

$$
\begin{gathered}
{ }_{0}^{t} \mathbf{B}_{L}={ }_{0}^{t} \mathbf{B}_{L 0}+{ }_{0}^{t} \mathbf{B}_{L 1} \\
{ }_{0}^{t} \mathbf{B}_{L 0}=\left[\begin{array}{ccccccccc}
{ }_{0} h_{1,1} & 0 & { }_{0} h_{2,1} & 0 & { }_{0} h_{3,1} & 0 & \cdots & { }_{0} h_{N, 1} & 0 \\
0 & { }_{0} h_{1,2} & 0 & { }_{0} h_{2,2} & 0 & { }_{0} h_{3,2} & \cdots & 0 & { }_{0} h_{N, 2} \\
{ }_{0} h_{1,2} & { }_{0} h_{1,1} & { }_{0} h_{2,2} & { }_{0} h_{2,1} & { }_{0} h_{3,2} & { }_{0} h_{3,1} & \cdots & { }_{0} h_{N, 2} & { }_{0} h_{N, 1} \\
\frac{h_{1}}{{ }^{0} \bar{x}_{1}} & 0 & \frac{h_{2}}{{ }^{0} \bar{x}_{1}} & 0 & \frac{h_{3}}{{ }^{0} \bar{x}_{1}} & 0 & \cdots & \frac{h_{N}}{{ }^{0} \bar{x}_{1}} & 0
\end{array}\right]
\end{gathered}
$$

where

$$
{ }_{0} h_{k, j}=\frac{\partial h_{k}}{\partial^{0} x_{j}} ; \quad u_{j}^{k}={ }^{i+\Delta t} u_{j}^{k}-{ }^{t} u_{j}^{k} ; \quad{ }^{0} \bar{x}_{1}=\sum_{k=1}^{N} h_{k}{ }^{0} x_{1}^{k} ; \quad N=\text { number of nodes }
$$

and

$$
\begin{aligned}
& { }_{0}^{1} \mathbf{B}_{L 1}=\left[\begin{array}{llll}
l_{110} h_{1,1} & l_{210} h_{1,1} & l_{110} h_{2,1} & l_{210} h_{2,1} \\
l_{120} h_{1,2} & l_{220} h_{1,2} & l_{120} h_{2,2} & l_{220} h_{2,2} \\
\left(l_{110} h_{1,2}+l_{120} h_{1,1}\right) & \left(l_{210} h_{1,2}+l_{220} h_{1,1}\right) & \left(l_{110} h_{2,2}+l_{120} h_{2,1}\right) & \left(l_{210} h_{2,2}+l_{220} h_{2,1}\right) \\
l_{33} \frac{h_{1}}{{ }_{0}} \bar{x}_{1} & 0 & l_{33 \frac{0}{0} \overline{x_{1}}} & 0
\end{array}\right. \\
& \ldots l_{11} h_{N, 1} \quad l_{210} h_{N, 1} \\
& \ldots l_{12} \mathrm{o}_{N, 2} \quad l_{22} \mathrm{o}_{\mathrm{N}, 2} \\
& \ldots \quad\left(l_{110} h_{N, 2}+l_{120} h_{N, 1}\right) \quad\left(l_{210} h_{N, 2}+l_{220} h_{N, 1}\right) \\
& \ldots \quad l_{33} \frac{h_{N}}{0_{\bar{x}} \bar{x}_{1}}
\end{aligned}
$$


Table I-continued

where

$$
\begin{aligned}
& l_{11}=\sum_{k=1}^{N}{ }_{0} h_{k, 1}{ }^{t} u_{1}^{k} ; \quad l_{22}=\sum_{k=1}^{N}{ }_{0} h_{k, 2} u_{2}^{k} ; \quad l_{21}=\sum_{k=1}^{N}{ }_{0} h_{k, 1}{ }^{t} u_{2}^{k} ; \\
& l_{12}=\sum_{k=1}^{N}{ }_{0} h_{k, 2}{ }^{t} u_{1}^{k} ; \quad l_{33}=\left(\sum_{k=1}^{N} h_{k}{ }^{t} u_{1}^{k}\right) /{ }^{0} \bar{x}_{1}
\end{aligned}
$$

Non-linear strain-displacement transformation matrix

$$
{ }_{0}^{1} \mathbf{B}_{N L}=\left[\begin{array}{ccccccccc}
{ }_{0} h_{1,1} & 0 & { }_{0} h_{2,1} & 0 & { }_{0} h_{3,1} & 0 & \cdots & { }_{0} h_{N, 1} & 0 \\
{ }_{0} h_{1,2} & 0 & { }_{0} h_{2,2} & 0 & { }_{0} h_{3,2} & 0 & \cdots & { }_{0} h_{N, 2} & 0 \\
0 & { }_{0} h_{1,1} & 0 & { }_{0} h_{2,1} & 0 & { }_{0} h_{3,1} & \cdots & 0 & { }_{0} h_{N, 1} \\
0 & { }_{0} h_{1,2} & 0 & { }_{0} h_{2,2} & 0 & { }_{0} h_{3,2} & \cdots & 0 & { }_{0} h_{N, 2} \\
\frac{h_{1}}{{ }^{0} \bar{x}_{1}} & 0 & \frac{h_{2}}{{ }^{0} \bar{x}_{1}} & 0 & \frac{h_{3}}{{ }^{0} \bar{x}_{1}} & 0 & \cdots & \frac{h_{N}}{0} \bar{x}_{1} & 0
\end{array}\right]
$$

2nd Piola-Kirchhoff stress matrix and vector

$$
{ }_{0}^{t} \mathbf{S}=\left[\begin{array}{ccccc}
{ }_{0}^{t} S_{11} & { }_{0}^{t} S_{12} & 0 & 0 & 0 \\
{ }_{0}^{t} S_{21} & { }_{0}^{t} S_{22} & 0 & 0 & 0 \\
0 & 0 & { }_{0}^{t} S_{11} & { }_{0}^{t} S_{12} & 0 \\
0 & 0 & { }_{0}^{t} S_{21} & { }_{0}^{t} S_{22} & 0 \\
0 & 0 & 0 & 0 & { }_{0}^{t} S_{33}
\end{array}\right] ; \quad{ }_{0}^{t} \hat{S}=\left[\begin{array}{c}
{ }_{0} S_{11} \\
{ }_{0}^{t} S_{22} \\
{ }_{0}^{t} S_{12} \\
{ }_{0}^{t} S_{33}
\end{array}\right]
$$

\section{Updated Lagrangian formulation}

Incremental strains

$$
\begin{aligned}
& { }_{t} \varepsilon_{11}={ }_{t} u_{1,1}+\frac{1}{2}\left[\left({ }_{t} u_{1,1}\right)^{2}+\left({ }_{t} u_{2,1}\right)^{2}\right] \\
& { }_{t} \varepsilon_{22}={ }_{t} u_{2,2}+\frac{1}{2}\left[\left({ }_{t} u_{1,2}\right)^{2}+\left({ }_{t} u_{2,2}\right)^{2}\right] \\
& { }_{t} \varepsilon_{12}=\frac{1}{2}\left[{ }_{t} u_{1,2}+{ }_{t} u_{2,1}\right]+\frac{1}{2}\left[{ }_{t} u_{1,1}{ }_{1,} u_{1,2}+{ }_{t} u_{2,1}, u_{2,2}\right] \\
& { }_{t} \varepsilon_{33}=\frac{u_{1}}{{ }^{t} x_{1}}+\frac{1}{2}\left(\frac{u_{1}}{{ }^{t} x_{1}}\right)^{2} \quad \text { (axisymmetric analysis) }
\end{aligned}
$$

where

$$
u_{i, j}=\frac{\partial u_{i}}{\partial^{t} x_{j}}
$$

\section{Linear strain-displacement transformation matrix}

Using

where

$$
{ }_{i} \mathbf{e}={ }_{i} \mathbf{B}_{L} \mathbf{u}
$$

$$
\begin{aligned}
& { }_{t} \mathbf{e}^{\mathrm{T}}=\left[{ }_{t} e_{11}, e_{22} 2_{1} e_{12,} e_{33}\right]: \quad \mathbf{u}^{\mathrm{T}}=\left[u_{1}^{1} u_{2}^{1} u_{1}^{2} u_{2}^{2} \ldots u_{1}^{N} u_{2}^{N}\right]
\end{aligned}
$$

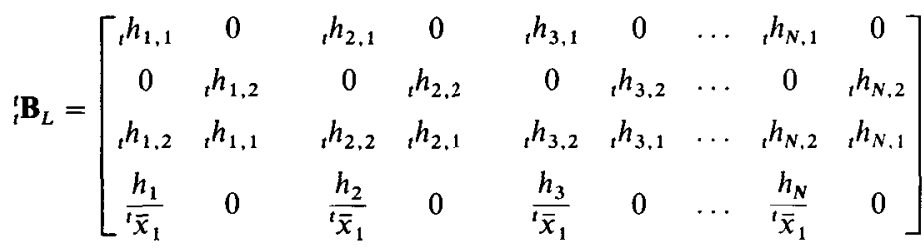


where

$$
{ }_{i} h_{k, j}=\frac{\partial h_{k}}{\partial^{t} x_{j}} ; \quad u_{j}^{k}={ }^{t+\Delta t} u_{j}^{k}-{ }^{t} u_{j}^{k} ; \quad ' \bar{x}_{1}=\sum_{k=1}^{N} h_{k}{ }^{t} x_{1}^{k} ; \quad N=\text { number of nodes }
$$

Non-linear strain-displacement transformation matrix

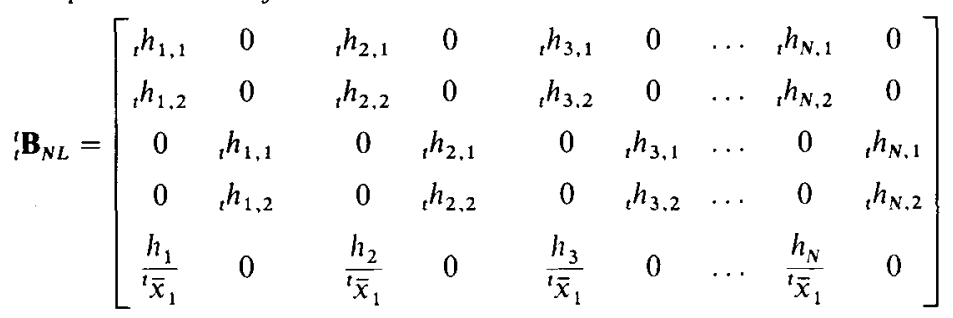

Cauchy stress matrix and stress vector

$$
{ }^{t} \tau=\left[\begin{array}{ccccc}
{ }^{t} \tau_{11} & { }^{t} \tau_{12} & 0 & 0 & 0 \\
{ }^{t} \tau_{21} & { }^{\prime} \tau_{22} & 0 & 0 & 0 \\
0 & 0 & { }^{t} \tau_{11} & { }^{t} \tau_{12} & 0 \\
0 & 0 & { }^{i} \tau_{21} & { }^{\prime} \tau_{22} & 0 \\
0 & 0 & 0 & 0 & { }^{t} \tau_{33}
\end{array}\right] ; \quad{ } \hat{\tau}=\left[\begin{array}{c}
{ }^{t} \tau_{11} \\
{ }^{t} \tau_{22} \\
{ }^{2} \tau_{12} \\
{ }^{\prime} \tau_{33}
\end{array}\right]
$$

and in the U.L. formulation this equation is

$$
\left({ }_{t}^{t} \mathbf{K}_{L}+{ }_{t}^{t} \mathbf{K}_{N L}\right) \mathbf{u}={ }^{t+\Delta t} \mathbf{R}-{ }_{i}^{t} \mathbf{F}-\mathbf{M}^{t+\Delta t}{ }_{\mathbf{i}}
$$

where ${ }^{t+\Delta t} t_{\mathbf{u}}$ is a vector of the element nodal point accelerations at time $t+\Delta t$, and $\mathbf{M}$ is the element mass matrix calculated at time 0 . In integrations (38) and (39), damping effects defined by a matrix $\mathbf{C}$ have been ignored. ${ }^{4}$

\section{Equilibrium iteration}

It is important to realize that equations (38) and (39) are only approximations to the actual equations to be solved in each time step, i.e. equations (7) and (16), respectively. Depending on the non-linearities in the system, the linearization of equations (15) and (23) may introduce errors which ultimately result into solution instability. For this reason it may be necessary to iterate in each load step until, within the necessary assumptions on the variation of the material constants and the numerical time integration, equations (7) and (16) are satisfied to a required tolerance. The equation used in the T.L. formulation is

$$
\left({ }_{0}^{t} \mathbf{K}_{L}+{ }_{0}^{t} \mathbf{K}_{N L}\right) \Delta \mathbf{u}^{(i)}={ }^{t+\Delta t} \mathbf{R}-{ }_{0}^{t+\Delta t} \mathbf{F}^{(i-1)}-\mathbf{M}^{t+\Delta t_{\mathbf{i}}}{ }^{(i)} \quad i=1,2,3 \ldots
$$

where ${ }^{t+\Delta t} \mathbf{u}^{(i)}={ }^{t+\Delta t} \mathbf{u}^{(i-1)}+\Delta \mathbf{u}^{(i)}$.

It should be noted that for $i=1$ equation (40) corresponds to equation (38), i.e. $\Delta \mathbf{u}^{(1)}=\mathbf{u}$, ${ }^{t+\Delta t} \dot{\mathbf{u}}^{(1)}={ }^{t+\Delta t} \dot{\mathbf{u}},{ }^{t+\Delta t} \mathbf{u}^{(0)}={ }^{t} \mathbf{u}$, and ${ }^{t+\Delta t} \mathbf{F}^{(0)}={ }_{0}^{t} \mathbf{F}$.

The calculation of the acceleration approximation ${ }^{t+\Delta t_{i}}{ }^{(i)}$ depends on the time integration scheme used.

The vector of nodal point forces equivalent to the element stresses, ${ }^{t+\Delta t} \mathbf{F}^{(i)}$, is the finite element evaluation of $\int_{O_{V}}{ }^{t+\Delta t} S_{0 i j}^{(i)} \delta^{t+\Delta t}{ }_{0} \varepsilon_{i j}^{(i)}{ }^{0} \mathrm{~d} v$, where the superscript $(i)$ shows that stresses and strains are evaluated using ${ }^{t+\Delta t} \mathbf{u}^{(i)}$. Since $\delta^{t+\Delta t} \varepsilon_{0} \varepsilon_{i j}=\frac{1}{2}\left(\delta_{0} u_{i, j}+\delta_{0} u_{j, i}+{ }^{t+\Delta t} u_{k, i} \delta_{0} u_{k, j}+{ }^{t+\Delta t} u_{k, j} \delta_{0} u_{k, i}\right)$, the 
nodal point forces are

$$
{ }^{t+\Delta t} \mathbf{F}^{(i)}=\int_{{ }^{0} V}{ }^{t+\Delta t}{ }_{0} \mathbf{B}_{L}^{(i)}{ }^{\mathbf{T}}+{ }_{0}^{t} \hat{\mathbf{S}}^{(i)}{ }^{0} \mathrm{~d} v
$$

in which the matrices ${ }^{t+\Delta t}{ }_{0}^{t} \mathbf{B}_{L}^{(i)}$ and ${ }^{t+}{ }_{0}^{\Delta t} \hat{\mathbf{S}}^{(i)}$ correspond to the matrices ${ }_{0}^{t} \mathbf{B}_{L}$ and ${ }_{0}^{t} \hat{\mathbf{S}}$ in Table I, but are defined for time $t+\Delta t$ and iteration $(i)$, respectively.

In the U.L. formulation the equation used for a single element with equilibrium iteration is

$$
\left.{ }_{t}^{t} \mathbf{K}_{L}+{ }_{t}^{t} \mathbf{K}_{N L}\right) \Delta \mathbf{u}^{(i)}={ }^{t+\Delta t} \mathbf{R}-{ }_{t+\Delta t}^{t+\Delta t} \mathbf{F}^{(i-1)}-\mathbf{M}^{t+\Delta t}{ }^{i}{ }^{(i)} \quad i=1,2,3, \ldots
$$

in which the $i$ th displacement and acceleration approximations are calculated as above and $\underset{t+\Delta t}{t+\Delta t} F^{(i)}$ is the finite element evaluation of

$$
\int_{t+\Delta t V^{(i)}}{ }^{t+\Delta t} \tau_{i j}^{(i)} \delta_{t+\Delta t} e_{i j}^{(i)}{ }^{t+\Delta t} \mathrm{~d} v^{(i)} ; \quad \text { i.e. } \quad{ }_{t+\Delta t}^{t+\Delta t} \mathbf{F}^{(i)}=\int_{t+\Delta t V^{(i)}}{ }^{t+\Delta t}{ }_{t} \mathbf{B}_{L}^{(i)^{\mathrm{T}}}{ }^{t+\Delta t} \hat{\tau}^{(i)}{ }^{t+\Delta t} \mathrm{~d} v^{(i)}
$$

where ${ }_{t+\Delta t}^{t+\Delta t} \mathbf{B}_{L}^{(i)}$ and ${ }^{t+\Delta t} \hat{\tau}^{(i)}$ correspond to the matrices ${ }_{t}^{t} \mathbf{B}_{L}$ and ${ }^{t} \hat{\tau}$ in Table I, respectively, but are defined for time $t+\Delta t$ and iteration (i), respectively.

It may be noted that the equilibrium iterations correspond to a modified Newton iteration within each load step. ${ }^{43}$ Table II summarizes the step-by-step algorithm used. For details on the Wilson $\theta$ and Newmark integration schemes see References 4, 5, 31 .

Table II. Summary of step-by-step integration

Initial calculations

1. Form mass matrix $\mathbf{M}$; initialize ${ }^{0} \mathbf{u},{ }^{0} \dot{\mathbf{u}},{ }^{0} \mathbf{u}$

2. Calculate the following constants:

tol $\leqslant 0.01 ; \quad$ nitem $\geqslant 3 ; \quad$ in static analysis $\theta=1$ and go to $A$.

Wilson $\theta$ method : $\theta \geqslant 1 \cdot 37$, usually $\theta=1 \cdot 4, \tau=\theta \Delta t$

$$
\begin{array}{lll}
a_{0}=6 / \tau^{2} & a_{1}=6 / \tau & a_{2}=2 \\
a_{3}=a_{0} / \theta & a_{4}=-a_{1} / \theta & a_{5}=1-3 / \theta \\
a_{6}=\Delta t / 2 & a_{7}=\Delta t^{2} / 6 &
\end{array}
$$

Newmark method : $\theta=1 \cdot 0, \delta \geqslant 0.50, \alpha \geqslant 0.25(0.5+\delta)^{2}, \tau=\Delta t$

$$
\begin{array}{lll}
a_{0}=1 /\left(\alpha \Delta t^{2}\right) & a_{1}=1 /(\alpha \Delta t) & a_{2}=1 /(2 \alpha)-1 \\
a_{3}=a_{0} & a_{4}=-a_{1} & a_{5}=-a_{2} \\
a_{6}=\Delta t(1-\delta) & a_{7}=\delta \Delta t &
\end{array}
$$

3. Calculate mass contribution to effective stiffness matrix : $\hat{\mathbf{K}}=a_{0} \mathbf{M}$

For each time step

A. Calculation of Displacement increment

(i) If a new stiffness matrix is to be formed, calculate and triangularize ${ }^{~} \hat{\mathbf{R}}$ :

$$
{ }^{t} \hat{\mathbf{K}}={ }^{t} \mathbf{K}+\hat{\mathbf{K}} ; \quad{ }^{t} \hat{\mathbf{K}}=\mathbf{L D L}^{\mathrm{T}}
$$

(ii) Form effective load vector:

$$
\left.{ }^{t+}{ }^{\tau} \hat{\mathbf{R}}={ }^{t} \mathbf{R}+\theta{ }^{t+\Delta t} \mathbf{R}-{ }^{t} \mathbf{R}\right)+\mathbf{M}\left(a_{1}{ }^{t} \dot{\mathbf{u}}+a_{2}{ }^{t}{ }^{i} \mathbf{u}\right)-{ }^{t} \mathbf{F}
$$


Table II-continued

(iii) Solve for displacement increments using latest $\mathbf{D}, \mathbf{L}$ factors:

$$
\mathbf{L D L}^{\mathrm{T}} \mathbf{u}={ }^{t+\tau} \hat{\mathbf{R}}
$$

(iv) If required, iterate for dynamic equilibrium; then initialize $\mathbf{u}^{(0)}=\mathbf{u}, i=0$

(a) $i=i+1$

(b) Calculate $(i-1)$ st approximation to accelerations and displacements :

$$
{ }^{t+} \tau_{\mathbf{i}} \mathbf{u}^{(i-1)}=a_{0} \mathbf{u}^{(i-1)}-a_{1}{ }^{\prime} \dot{\mathbf{u}}-a_{2}{ }^{t} \mathbf{i} ; \quad{ }^{t+}{ }^{t} \mathbf{u}^{(i-1)}={ }^{i} \mathbf{u}+\mathbf{u}^{(i-1)}
$$

(c) Calculate $(i-1)$ st effective out-of-balance loads:

$$
{ }^{t+\tau} \hat{\mathbf{R}}^{(i-1)}={ }^{t} \mathbf{R}+\theta\left({ }^{t+\Delta t} \mathbf{R}-{ }^{t} \mathbf{R}\right)-\mathbf{M}^{t+\tau_{\mathbf{u}}}{ }^{(i-1)}-{ }^{t+\tau} \mathbf{F}^{(i-1)}
$$

(d) Solve for $i$ th correction to displacement increments :

$$
\operatorname{LDL}^{\mathbf{T}} \Delta \mathbf{u}^{(i)}={ }^{++} \hat{\mathbf{R}}^{(i-1)}
$$

(e) Calculate new displacement increments:

$$
\mathbf{u}^{(i)}=\mathbf{u}^{(i-1)}+\Delta \mathbf{u}^{(i)}
$$

(f) Iteration convergence if $\left\|\Delta \mathbf{u}^{(i)}\right\|_{2} /\left\|\mathbf{u}^{(i)}+\mathbf{u}\right\|_{2}<$ tol

If convergence : $\mathbf{u}=\mathbf{u}^{(i)}$ and go to $B$;

If no convergence and $i<$ nitem: go to (a); otherwise restart using new stiffness matrix and/or a smaller time step size.

B. Calculate new accelerations, velocities and displacements

Wilson $\theta$ method :

$$
\begin{aligned}
& { }^{t+}+\Delta^{t} \mathbf{i}=a_{3} \mathbf{u}+a_{4}{ }^{t} \dot{\mathbf{u}}+a_{5}{ }^{{ }} \mathbf{i} \\
& { }^{t+\Delta t} \dot{\mathbf{u}}={ }^{\prime} \dot{\mathbf{u}}+a_{6}\left({ }^{t+\Delta t} \dot{\mathbf{u}}+{ }^{t} \dot{\mathbf{u}}\right) \\
& { }^{t+\Delta t} \mathbf{u}={ }^{t} \mathbf{u}+\Delta t^{t} \dot{\mathbf{u}}+a_{7}\left({ }^{t+\Delta t} \ddot{\mathbf{u}}+2{ }^{t} \dot{\mathbf{u}}\right)
\end{aligned}
$$

Newmark method:

$$
\begin{aligned}
& { }^{t+\Delta t} \dot{\mathbf{u}}=a_{3} \mathbf{u}+a_{4}{ }_{4} \dot{\mathbf{u}}+a_{5}{ }_{5} \dot{\mathbf{u}} \\
& { }^{t+\Delta t} \dot{\mathbf{u}}={ }^{t} \dot{\mathbf{u}}+a_{6}{ }^{t} \ddot{\mathbf{u}}+a_{7}{ }^{t+\Delta t^{\prime} \dot{\mathbf{u}}} \\
& { }^{t+\Delta t} \mathbf{u}={ }^{t} \mathbf{u}+\mathbf{u}
\end{aligned}
$$

\section{CONSTITUTIVE RELATIONS}

An important aspect in the solution of non-linear problems is the calculation of the constitutive tensors, which define the stress-strain matrices in the finite element evaluations. In the isoparametric finite element discretization it is necessary to evaluate the stress-strain matrices at the element integration points, and they are required for the calculation of the element stiffness matrices and stress vectors.

\section{Linear elasticity and hyperelasticity}

Elastic and hyperelastic materials are relatively easy to deal with in practical analyses. In the T.L. formulation the stress-strain relations are ${ }^{11}$

$$
{ }_{0}^{t} S_{i j}={ }_{0}^{t} C_{i j r s}{ }_{0}^{t} \varepsilon_{r s}
$$

where ${ }_{0}^{t} S_{i j}$ is the 2 nd Piola-Kirchhoff stress tensor, ${ }_{0}^{t} \varepsilon_{r s}$ is the Green-Lagrange strain tensor and ${ }_{0}^{t} C_{i j r s}$ is the material property tensor in the configuration at time $t$. The relation in equation (44) 
can be written for all configurations at time $0, \Delta t, 2 \Delta t, \ldots$. In the U.L. formulation the constitutive relation equivalent to equation (44) is

$$
{ }^{t} \tau_{i j}={ }_{i}^{t} C_{i j r s}{ }^{t} \varepsilon_{r s}
$$

in which ${ }^{t} \tau_{i j}$ is the Cauchy stress tensor, ${ }^{\prime} \varepsilon_{r s}$ is the Almansi strain tensor and ${ }_{i}^{t} C_{i j r s}$ is the material property tensor at time $t$.

Considering linear elasticity ${ }_{0}^{t} C_{i j r s}$ and ${ }_{i}^{t} C_{i j r s}$ are both constant and defined in terms of the Young's moduli and Poisson's ratios of the material. However, it should be noted that specifying constant ${ }_{t}^{t} C_{i j r s}$ is equivalent to using a material tensor ${ }_{0}^{t} C_{i j r s}$, which is deformation dependent, and vice versa; namely the following relations exist

$$
\begin{aligned}
& { }_{0}^{t} C_{m n p q}=\frac{{ }^{0} \rho}{{ }^{t} \rho}{ }_{t}^{0} x_{m, i}{ }_{t}^{0} x_{n, j}{ }_{t}^{t} C_{i j r s}{ }_{t}^{0} x_{p, r}{ }_{t}^{0} x_{q, s} \\
& { }_{t}^{t} C_{m n p q}=\frac{{ }^{t} \rho}{{ }^{0} \rho}{ }_{0}^{t} x_{m, i}{ }_{0}^{t} x_{n, j}{ }_{0}^{t} C_{i j r s}{ }_{0}^{t} x_{p, r}{ }_{0}^{t} x_{q, s}
\end{aligned}
$$

The constitutive relations in equations (44) and (45) are used in the evaluation of the element stress matrices and stress vectors (see Table I), i.e. total 2nd Piola-Kirchhoff and Cauchy stresses are calculated directly from total Green-Lagrange and Almansi strains, respectively. However, in the calculation of the linear strain stiffness matrices at time $t$, tangent material property tensors are required. In the T.L. and U.L. formulations the relations considered are ${ }_{0} S_{i j}={ }_{0} C_{i j r s} \varepsilon_{r s}$ and ${ }_{t} S_{i j}={ }_{t} C_{i j r s t} \varepsilon_{r s}$, respectively, in which, for linear elasticity,

$$
\begin{aligned}
& { }_{0} C_{i j r s}={ }_{0}^{t} C_{i j r s} \\
& { }_{t} C_{i j r s}={ }_{i}^{t} C_{i j r s}
\end{aligned}
$$

Considering hyperelasticity the stress-strain relations are derived from the strain energy function. ${ }^{11,19,33}$ In this study the constitutive relations defining ${ }_{0}^{t} S_{i j}$ and ${ }_{0} C_{i j r s}$ in terms of the Green-Lagrange strain at time $t$ for a rubber-like material in plane stress conditions have been derived. ${ }^{4,19}$ Therefore, to use the U.L. formulation, it is necessary to transform ${ }_{0}^{t} S_{i j}$ and ${ }_{0} C_{i j r s}$ to ${ }^{\prime} \tau_{i j}$ and ${ }_{t} C_{i j r s}$, respectively, as expressed in equations (5) and (47).

It is important to note that in the analysis of elastic and hyperelastic materials identical numerical results are obtained using the T.L. and U.L. formulations provided the material tensors are related as given in equations (46) and (47). Also, since the material constants are independent of the history of solution, analysis errors result only from the isoparametric finite element formulation and the time integration scheme, provided equilibrium iterations are performed. Therefore, in the analysis of elastic and hyperelastic materials the analysis errors are quite similar to those in small displacement linear elastic analysis.

\section{Hypoelasticity including elastoplasticity}

For hypoelastic materials the constitutive tensors relate increments in stresses to increments in deformations. ${ }^{11}$ Since the constitutive relations depend, in general, on the stress and strain history, the use of a material law corresponding to the T.L. or the U.L. formulation must depend to a large degree on the possibility of performing experiments to obtain the appropriate material constants. In this context it should be noted that a great deal of additional research is still required to formulate and evaluate appropriate material constants for hypoelastic materials, in particular, for the identification of large strain behaviour. ${ }^{19,22,23}$ Although the formulations presented below are applicable to large strain conditions, in actual practical analysis the material law is 
most likely to be defined only for small strains. ${ }^{4}$ An important such case here included is the elastic-plastic material behaviour characterized using the flow theory, which can be used in the analysis of large displacement but small strain problems.

Using the T.L. formulation, hypoelastic material behaviour can be described using equation (14), i.e.

$$
{ }_{0} S_{i j}={ }_{0} C_{i j r s} \varepsilon_{r s}
$$

in which ${ }_{0} C_{i j r s}$ depends on the history of the Green-Lagrange strains and 2nd Piola-Kirchhoff stresses. The stresses at time $t+\Delta t$ are calculated using equation (9), i.e.

$$
{ }_{0}^{t+\Delta t} S_{i j}={ }_{0}^{t} S_{i j}+{ }_{0} S_{i j}
$$

In the analysis using equations (14) and (9) it is assumed that the material tensor ${ }_{0} C_{i j r s}$ is evaluated in the same way as in small displacement analysis, but the stress and strain variables of the T.L. formulation are used to define the history of the material. A main advantage of adopting this material description is that it is relatively simple to use. Namely, assume that a subroutine to calculate the material law in small displacement analysis has been written; then the same program would also calculate ${ }_{0} C_{i j r s}$ in large displacement analysis by simply using GreenLagrange strains and 2nd Piola-Kirchhoff stresses to define the stress and strain history. ${ }^{10,22}$

Similarly to equation (14), in the U.L. formulation hypoelastic material behaviour may be described using equation (22), i.e.

$$
{ }_{t} S_{i j}={ }_{t} C_{i j r s t} \varepsilon_{r s}
$$

in which ${ }_{t} C_{i j r s}$ is defined by the history of Cauchy stresses and the accumulation of the instantaneous plastic strain increments. The constitutive relation in equation (22) may be more appealing than the T.L. material law in equation (14) since physical stress components are used to define the material constants, and ${ }_{t} \varepsilon_{r s}$ approximated by ${ }_{t} e_{r s}$ can kinematically be understood to be the addition of elastic and plastic strain increments, just as in small displacement analysis. Having calculated $S_{i j}$ from the relation ${ }_{t} S_{i j}=C_{i j r s} e_{r s}$, the Cauchy stresses at time $t+\Delta t$ are obtained using equation (17), i.e.

$$
{ }^{t+\Delta t} S_{i j}={ }^{t} \tau_{i j}+{ }_{t} S_{i j}
$$

and the transformation

$$
{ }^{t+\Delta t} \tau_{s r}=\frac{{ }^{t+\Delta t} \rho}{{ }^{t} \rho}{ }^{t+\Delta t}{ }_{t} x_{s, i}{ }^{t+\Delta t}{ }_{t} S_{i j}{ }^{t+\Delta t}{ }_{t} x_{r, j}
$$

A third possibility is to characterize the material behaviour using a stress rate which is defined with respect to the current moving co-ordinates within the time interval $t$ to $t+\Delta t{ }^{11,15,16,23}$ The stress rate used must be invariant with respect to rigid body rotations, and one possibility is to use the Jaumann stress rate, which, at time $t$, is defined as

$$
{ }^{t} \tau_{i j}^{\nabla}=\frac{\mathrm{D}}{\mathrm{D} t} \tau_{i j}-{ }^{t} \tau_{i p}{ }^{t} \Omega_{p j}-{ }^{t} \tau_{j p}{ }^{t} \Omega_{p i}
$$

where $\mathrm{D} / \mathrm{D} t$ denotes time derivative with ${ }^{t} x_{i}, i=1,2,3$, kept constant,

$$
{ }^{t} \tau_{i j}^{\nabla}={ }_{t} C_{i j r s} \frac{\mathrm{D}}{\mathrm{D} t} e^{e} e_{r s}
$$


and ${ }^{t} \Omega_{p j}$ are the Cartesian components of the spin tensor,

$$
{ }^{t} \Omega_{p j}=\frac{1}{2} \frac{\mathrm{D}}{\mathrm{D} t}\left({ }_{t} u_{j, p}-{ }_{t} u_{p, j}\right)
$$

Equations (51) to (53) need to be considered in the evaluation of the tangent stiffness matrix and in the calculation of the current stress conditions. The constitutive tensor relating the Jaumann stress rate tensor ${ }^{t} \tau_{i j}^{\nabla}$ to the incremental strain rate tensor $(\mathrm{D} / \mathrm{D} t)_{t} e_{r s}$ is calculated in the same way as in small displacement analysis, but using Cauchy stresses to define the history of the material. In the evaluation of the linear strain tangent stiffness matrix, instead of equation (22), the following approximate relation may be used,

$$
D^{t} \tau_{i j}={ }_{t} C_{i j r s} e_{r s}
$$

where $D$ signifies 'discrete increment in', and therefore $D_{t} e_{r s} \equiv{ }_{t} e_{r s}$. Considering the calculation of Cauchy stresses at time $t+\Delta t,{ }^{t+\Delta t} \tau_{i j}$, it is important to use equations (51) to (53) in small enough increments of time. In elastic-plastic analysis it is in any case necessary to evaluate the stress increments by numerical integration of the elastic-plastic material law times the strain increments, and it is efficient to include equation (51) in this integration.

In this study the T.L. material description using equations (14) and (9) and the U.L. material descriptions given in equations (22), (17), (50) and in equations (51) to (54) have been implemented. The U.L. formulations will be referred to as U.L. with transformation, U.L.(T), and U.L. with Jaumann stress rate, U.L. (J), respectively. The calculation of the elastic-plastic material constants and stress vectors in the T.L., U.L.(T) and U.L.(J) formulations is presented in detail in Reference 4.

In the above enumeration it was assumed that the solution procedure, namely the T.L. or U.L. formulation, is chosen according to the definition of the constitutive tensor. Since the constitutive tensor is dependent on stress and strain quantities, it is expedient to use the specific incremental finite element formulation, for which the material law is defined. If this is not done, it is necessary to evaluate the required stress and strain quantities for the calculation of the material constants and transform the constitutive relations using equation (46) or equation (47).

\section{DEFORMATION DEPENDENT LOADING}

So far it has been assumed that the loads are independent of the configuration of the body. In practice, therefore, the external loads for each step can be calculated and stored on back-up storage before the actual time integration is carried out. However, when the structure undergoes large displacements or large strains it may be necessary to consider the externally applied loads to be configuration dependent.

An important type of loading, which may need to be considered as deformation dependent, is pressure loading. ${ }^{33}$ In this case the loading to be used in the T.L. formulation is

$$
{ }^{t+\Delta t} t_{k}{ }^{0} \mathrm{~d} a=-\frac{{ }^{0} \rho}{t+\Delta t \rho}{ }^{t+\Delta t} p_{t+\Delta t}{ }^{0} x_{i, k}{ }^{0} n_{i}{ }^{0} \mathrm{~d} a
$$

and in the U.L. formulation

$$
{ }^{t+\Delta t}{ }_{t} t_{k}{ }^{t} \mathrm{~d} a=-\frac{{ }^{t} \rho}{{ }^{t+\Delta t} \rho}{ }^{t+\Delta t} p_{t+\Delta t}{ }^{t} x_{i, k}{ }^{t} n_{i}{ }^{t} \mathrm{~d} a
$$

where ${ }^{\prime} n_{i}=$ component $i$ of the normal $n$ in the configuration at time $t$, and similarly for time 0 , and ${ }^{t+\Delta t} p=$ surface pressure in the configuration at time $t+\Delta t$. Equation (55) can be written 
in the form

$$
{ }^{t+\Delta t} t_{k}{ }^{0} \mathrm{~d} a=-\frac{{ }^{0} \rho}{t+\Delta t \rho}{ }^{t+\Delta t} p{ }_{t}^{0} x_{i, k}{ }^{0} n_{i}{ }^{0} \mathrm{~d} a+\frac{}{t+\Delta t} \rho_{t+\Delta t}{ }^{t+\Delta} p{ }_{t}^{0} x_{i, j} \quad t+\Delta t u_{j, k}{ }^{0} n_{i}{ }^{0} \mathrm{~d} a
$$

and similarly equation (56) becomes

$$
{ }^{t+\Delta t}{ }_{i} t_{k}{ }^{t} \mathrm{~d} a=-\frac{{ }^{t} \rho}{t+\Delta t \rho}{ }^{t+\Delta t} p^{t} n_{k}{ }^{t} \mathrm{~d} a+\frac{{ }^{t} \rho}{{ }^{t+\Delta t} \rho}{ }^{t+\Delta t} p_{t+\Delta t} u_{i, k}{ }^{t} n_{i}{ }^{t} \mathrm{~d} a
$$

where in both formulations the first integral enters the load vector and, assuming that ${ }_{t+\Delta t} u_{i, k} \doteq$ ${ }_{i} u_{i, k}$, the second integral contributes to the system tangent stiffness matrix. It should be noted that this is a non-symmetric contribution to the stiffness matrix, and is therefore, in practice, computationally inefficient to handle. Using equilibrium iterations, it appears more efficient, at least when pressures are reasonably small, to neglect the contribution of the pressure loads to the stiffness matrix. In the iteration the loads are then evaluated as

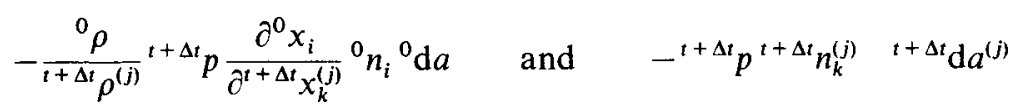

in the T.L. and U.L. formulations, respectively, where the right superscript $(j)$ indicates the configuration of the iteration. It is seen that although the same approximations are involved in both formulations, the U.L. formulation requires less numerical operations and seems more natural to use.

\section{SAMPLE SOLUTIONS}

All solutions presented in the following have been obtained using the algorithm presented in Table II, in which the selected parameters were tol $=0.001$, nitem $=15, \theta=1.4, \delta=0.50$ and $\alpha=0.25$. No attempt was made to optimize the solution times by selecting the most effective load step increments. In the dynamic analyses, nearly always one or two equilibrium iterations were sufficient in each time step and the computer time used was in all analyses rather small.

The time step used in an analysis is denoted by $\Delta t$ and was selected as a reasonable fraction of the fundamental period, $T_{f}$, of the structure at time 0 . In all dynamic analyses zero initial conditions on the displacements, velocities and accelerations were assumed.

For the finite element discretization 4- or 8-node two-dimensional elements have been employed (Figure 2). The material properties given have always been assumed to be defined corresponding to the specific formulation used for solution.

\section{Large displacement static and dynamic analysis of a cantilever}

The cantilever in Figure 3 under uniformly distributed load was analyzed using the T.L. and U.L. formulations. The cantilever was idealized using five 8-node plane stress elements.

Static solutions were obtained for the loading retaining its vertical direction, and for the loading remaining perpendicular to the top and bottom surfaces of the cantilever, i.e. deformation dependent follower loading. In the finite element solution the follower loading can be defined by specifying the direction of the nodal loads to pass through two nodal points, the co-ordinates of which are updated in each load step. In this specific analysis, the top and bottom surface nodal points of the cantilever have been used to define the direction of the loading. In addition to the U.L. and T.L. formulations also the U.L.(T) and U.L.(J) formulations have been used in the solution for deformation independent loading, in order to assess the accuracy that may be 


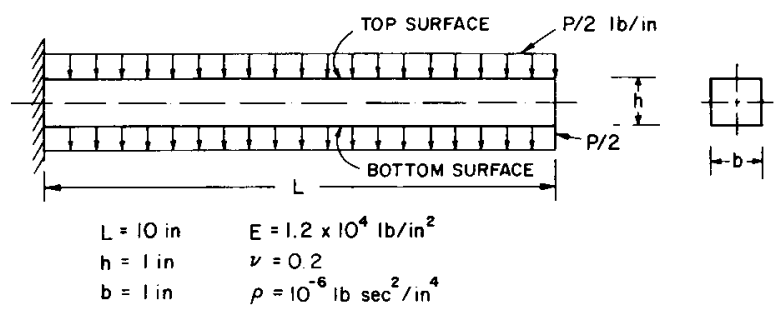

Figure 3. Cantilever under uniformly distributed load

expected in elastic-plastic analysis. It is seen that, to the precision possible to show in Figure 4, the T.L., U.L., U.L.(T) and U.L.(J) solutions predict the same response (although the same $E$ and $v$ have been used in each analysis, Figure 3) and that excellent agreement has been obtained with an analytical solution reported by Holden. ${ }^{17}$

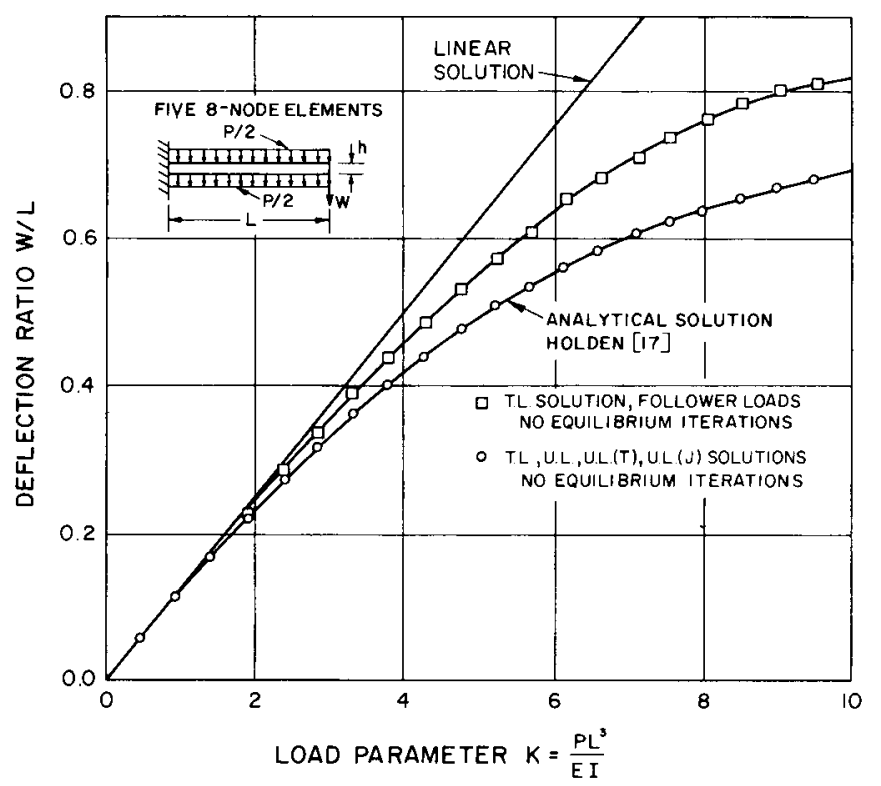

Figure 4. Large deflection analysis of cantilever under uniformly distributed load

For the dynamic analysis the T.L. formulation was selected. Figure 5 shows the results obtained using the Newmark integration scheme. It is seen that the solution predicted using a time step $\Delta t \cong T_{f} / 42$, where $T_{f}$ is the fundamental period of the cantilever, is significantly different from the solution obtained with the smaller time step $\Delta t \cong T_{f} / 126$, unless equilibrium iterations are used. An average of 4 iterations per time step were required. The analysis therefore shows the importance of using equilibrium iterations in the response calculations of this structure, unless a small time step, $\Delta t$, is used.

It should be noted that a main characteristic of the cantilever is that the structure stiffens with increasing displacement, which, as shown in Figure 5, results in a substantial decrease in amplitude and effective period of vibration. It is the stiffening of a structure that can result in convergence difficulties in equilibrium iteration. ${ }^{4}$ 


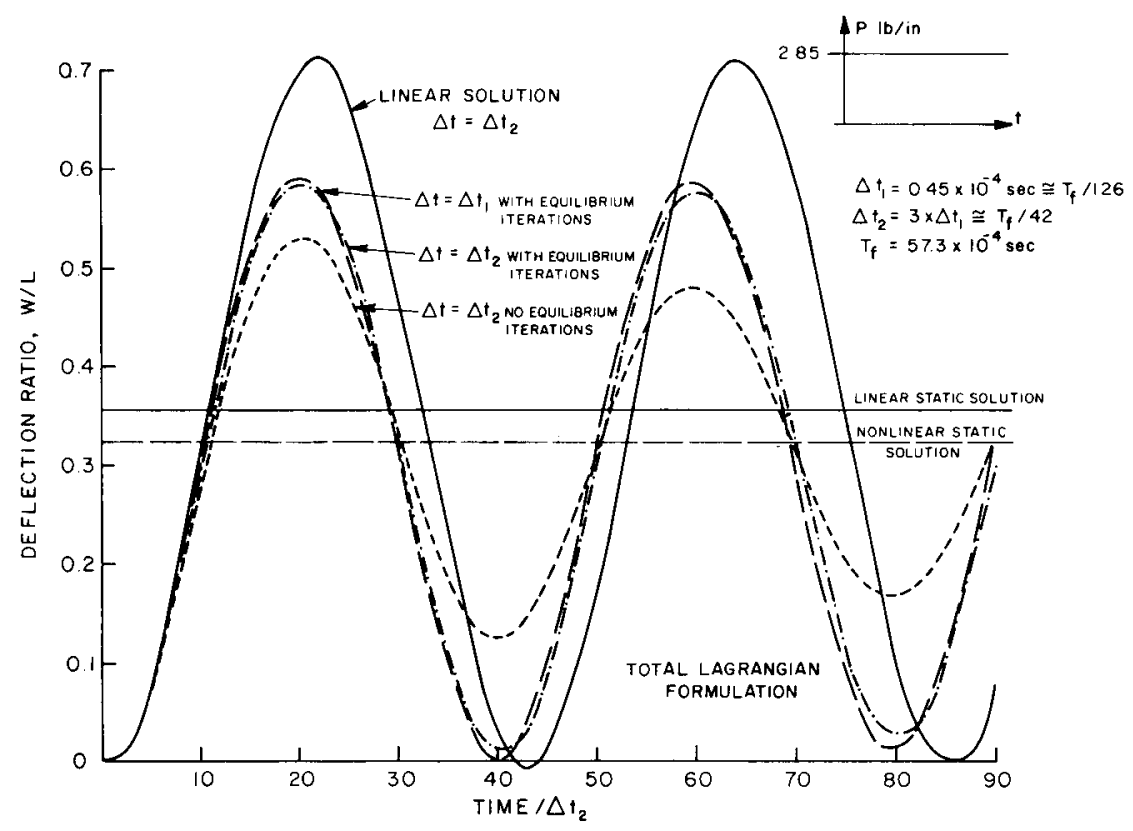

Figure 5. Large displacement dynamic response of cantilever under uniformly distributed load, Newmark method $\delta=0.50, \alpha=0.25$

Static large displacement analysis of a spherical shell

The clamped shallow spherical shell in Figure 6 subjected to uniform pressure was analyzed using a finite element idealization of eight 8-node elements.

Figure 6 shows the load deflection curve predicted using the T.L. formulation. In the analysis, 36 load steps with an average of about 3 to 4 equilibrium iterations in each step were used. The results are compared with an analytical solution of Kornishin and Isanbaeva, ${ }^{21}$ and a finite element solution of Yeh. ${ }^{42}$ As shown, good agreement between the different solutions has been obtained. Since equilibrium iterations were performed in the present solution, the oscillating behaviour at the beginning of the post-buckling range in Yeh's solution was not obtained.

The U.L. formulation gave almost indistinguishable results to those of the T.L. formulation.

Static and dynamic large displacement analysis of a second spherical shell

The spherical shell subjected to a concentrated apex load shown in Figure 7 was analyzed for static and dynamic response.

Figure 7 shows the static load-deflection response predicted in this study and by Stricklin ${ }^{37}$ and Mescall. ${ }^{27}$ Good agreement between the different solutions has been obtained. In the present solutions, the T.L. and U.L. formulations were used and no equilibrium iterations have been performed. In addition, to assess the accuracy that may be obtained in elastic-plastic analysis, the U.L.(T) and U.L.(J) formulations have been used. Figure 8 compares the T.L. response predictions with two U.L.(T) and U.L.(J) solutions. It is seen that the U.L.(T) and U.L.(J) solutions approach the T.L. solution as the load steps become smaller.

The dynamic response calculated using the Wilson $\theta$ integration method when the apex load is applied as a step load is shown in Figure 9. It is observed that for this problem the differences between the solutions using equilibrium iteration and not iterating for equilibrium are small. 


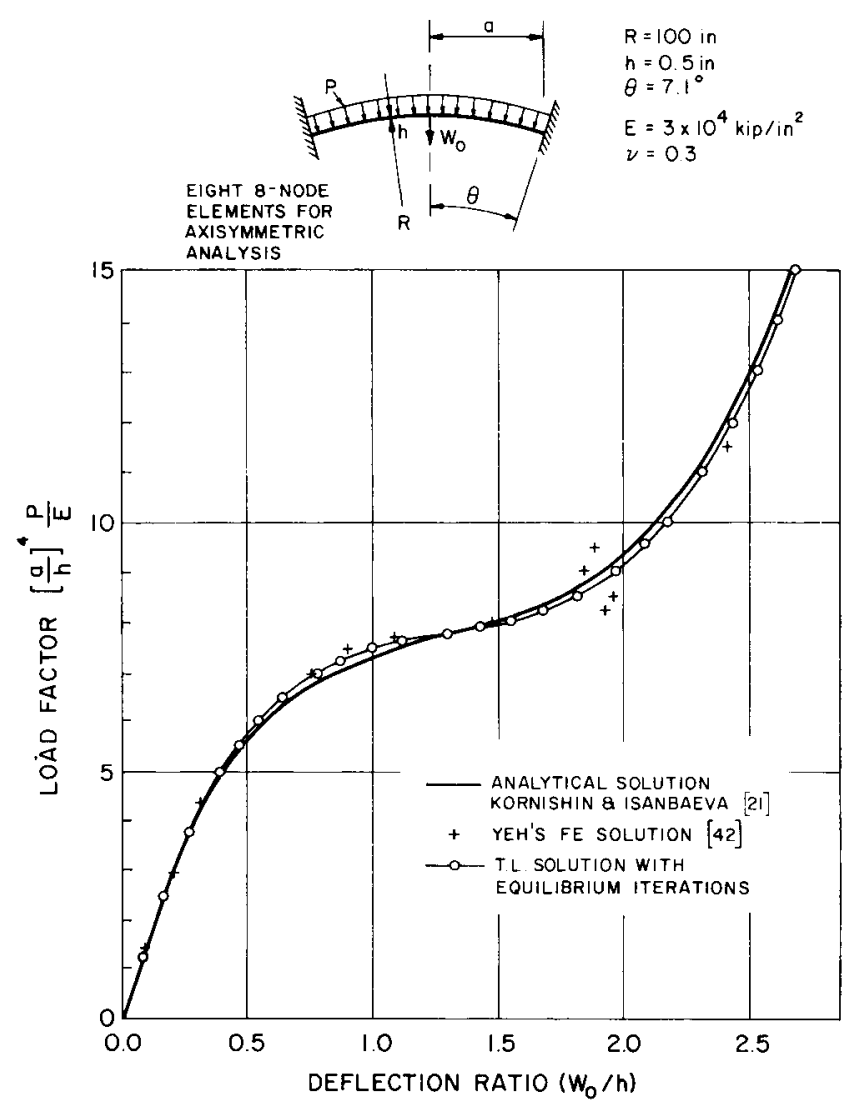

Figure 6. Load-deflection curve for a shallow spherical shell

The much larger response and effective period predicted in the non-linear analysis is a result of the softening behaviour of the structure with increasing load. It should be noted that in the analysis of this highly non-linear shell no difficulties were encountered using the Wilson or the Newmark integration methods, and practically identical results were obtained. ${ }^{4}$

\section{Large displacement static buckling analysis of an arch}

The clamped circular arch shown in Figure 10 was analyzed for buckling due to a single static load using the T.L. and U.L. formulations with equilibrium iterations.

Figure 10 shows the calculated load-deflection curve of the arch. The differences in the displacements calculated using the U.L. and T.L. formulations were less than two per cent. The solutions were obtained using 28 load steps with an average of two to three equilibrium iterations per step.

The same arch was also analyzed by Mallet and Berke, who used four 'equilibrium-based' elements. ${ }^{24}$ Dupuis and many others, analyzed the arch with curved beam elements, and used this example to demonstrate the convergence of their Lagrangian and 'updated' formulations. In the latter formulation only the nodal points were updated, but not the geometry within the elements. As shown in Figure 10, the results are very sensitive to the number of elements used and are not satisfactory. Dupuis and many others, also compared the calculated results with 


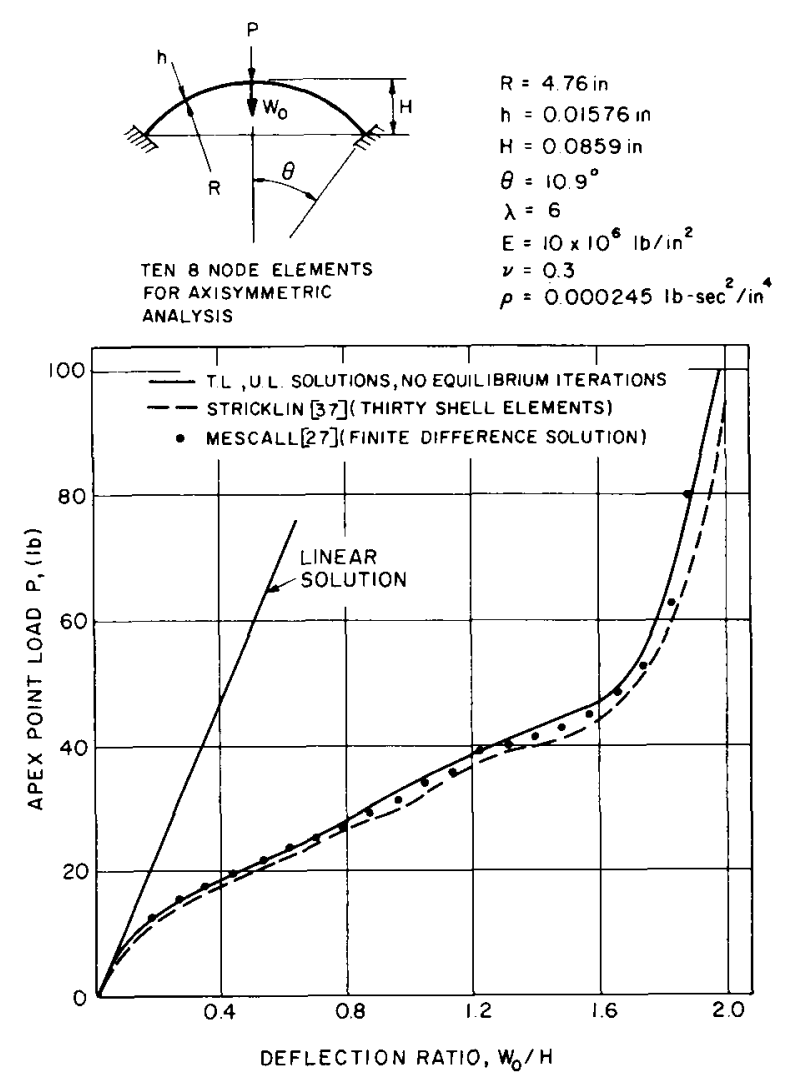

Figure 7. Load-deflection curves for spherical shell

experimental results by Gjelsvik and Bodner, ${ }^{12}$ whose predicted buckling load is about 10 per cent lower than that calculated by Mallet. However, it need be realized that an arch with a parameter $\lambda=11.6$ is already influenced by antisymmetric buckling modes, which, although possible in the experiment, have not been taken account of in the analyses. ${ }^{34}$ The results obtained in this study are therefore satisfactory.

\section{Elastic dynamic snap buckling of a second arch}

A dynamic buckling analysis of the circular arch shown in Figure 11 was carried out. The material of the arch was assumed to be isotropic linear elastic.

In the analyses the T.L. formulation was used. The uniformly distributed pressure load was applied as a step load. The time step to fundamental period ratio, $\Delta t / T_{f}$, was approximately $1 / 70$.

The arch is an example of Humphreys' analytical and experimental investigation, who solved the governing differential equation using an analogue computer. ${ }^{18}$ Humphreys concluded that the buckling load of this arch is not influenced by antisymmetric modes.

Figure 12 shows the displacement response predicted in this study using the Wilson $\theta$ integration scheme. The solution obtained by Humphreys is also shown. In the Figure, the deflection ratio $\Delta$ defined as

$$
\Delta=\frac{\text { average normal deflection } w}{\text { average rise of arch }=H / 2}
$$



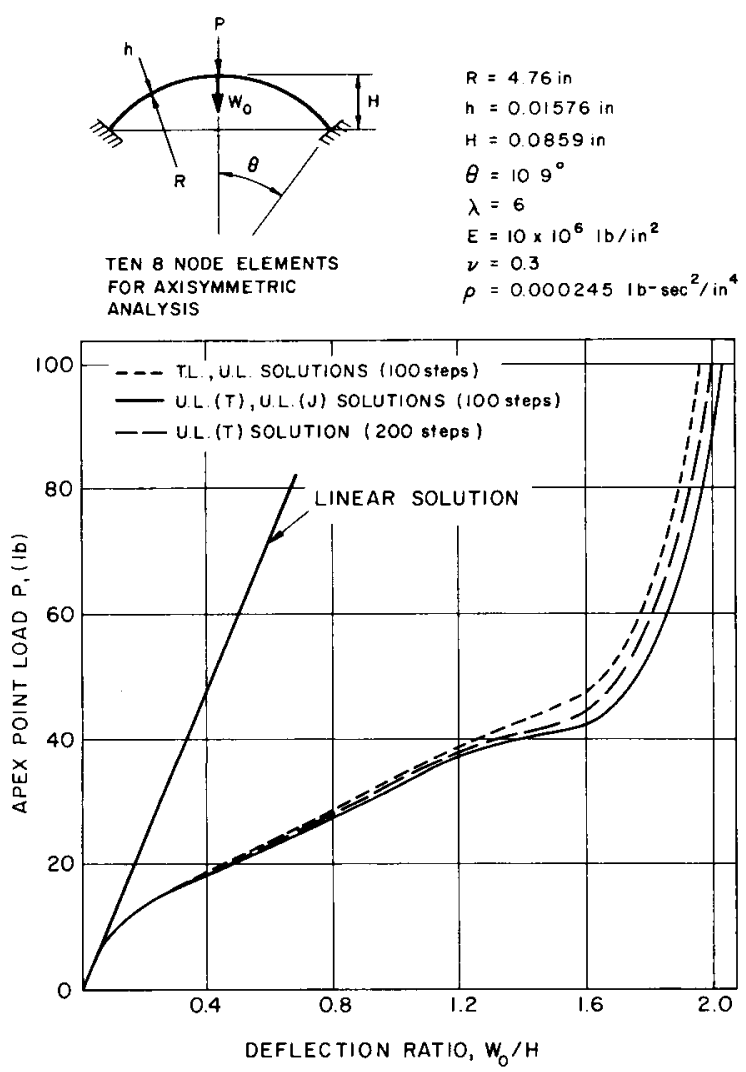

Figure 8. Load-deflection curves for spherical shell

is used. The dynamic buckling of the arch occurs at that load level at which a sudden increase in the deflection ratio $\Delta$ is measured. Figure 12 shows that at $p_{0}=0.190$ the arch oscillates about a position of approximately $\Delta=0.25$, and that at $p_{0}=0.200$ the arch first snaps through, and then oscillates about a position of approximately $\Delta=2.5$. Therefore, the buckling load predicted here lies between $p_{0}=0.190$ and $p_{0}=0.200$, which is about five per cent lower than that predicted by Humphreys.

It should be noted that for a load larger than the buckling load, i.e. for $p_{0}=0 \cdot 25$, the maximum response increases only little. The results for $p_{0}=0.250$ are in essential agreement with Humphreys' results, where the slightly larger response agrees with the observation that a smaller buckling load was predicted in this study. The discrepancies in the results can arise from approximations in either analysis: Humphreys' series solution is based on the assumption of shallowness, i.e. $q$ and $w$ are measured vertically, and in the series solution only a finite number of terms have been included.

It is noted that in a practical analysis damping should be included and a longer time range may be considered as well.

\section{Large displacement and large strain static and dynamic analysis of a rubber sheet with a hole}

A plane stress analysis of the rubber sheet shown in Figure 13 was carried out. The purpose of this analysis was to test the capability of predicting static and dynamic large strain response.

The material of the rubber sheet was assumed to be of Mooney-Rivlin type. The specific material constants used for the hyperelastic incompressible material were $C_{1}=25 \mathrm{psi}, C_{2}=$ 


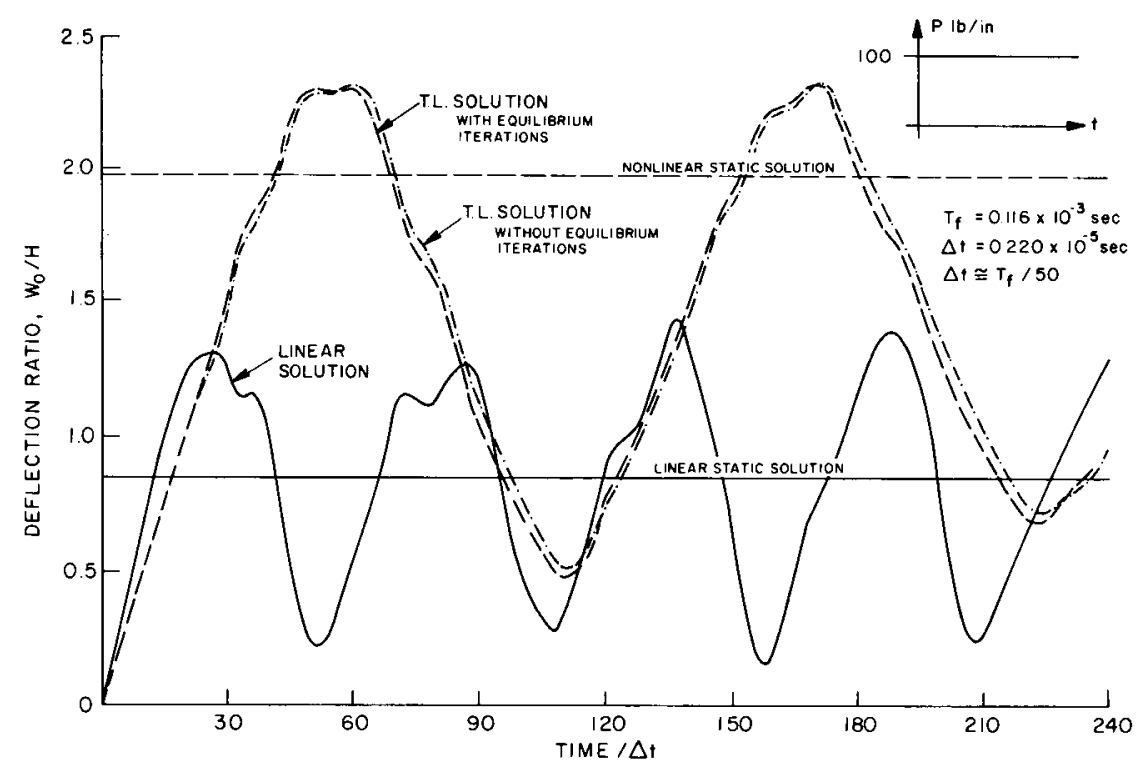

Figure 9. Non-linear dynamic response of spherical shell, Wilson $\theta$ method, $\theta=1.4$

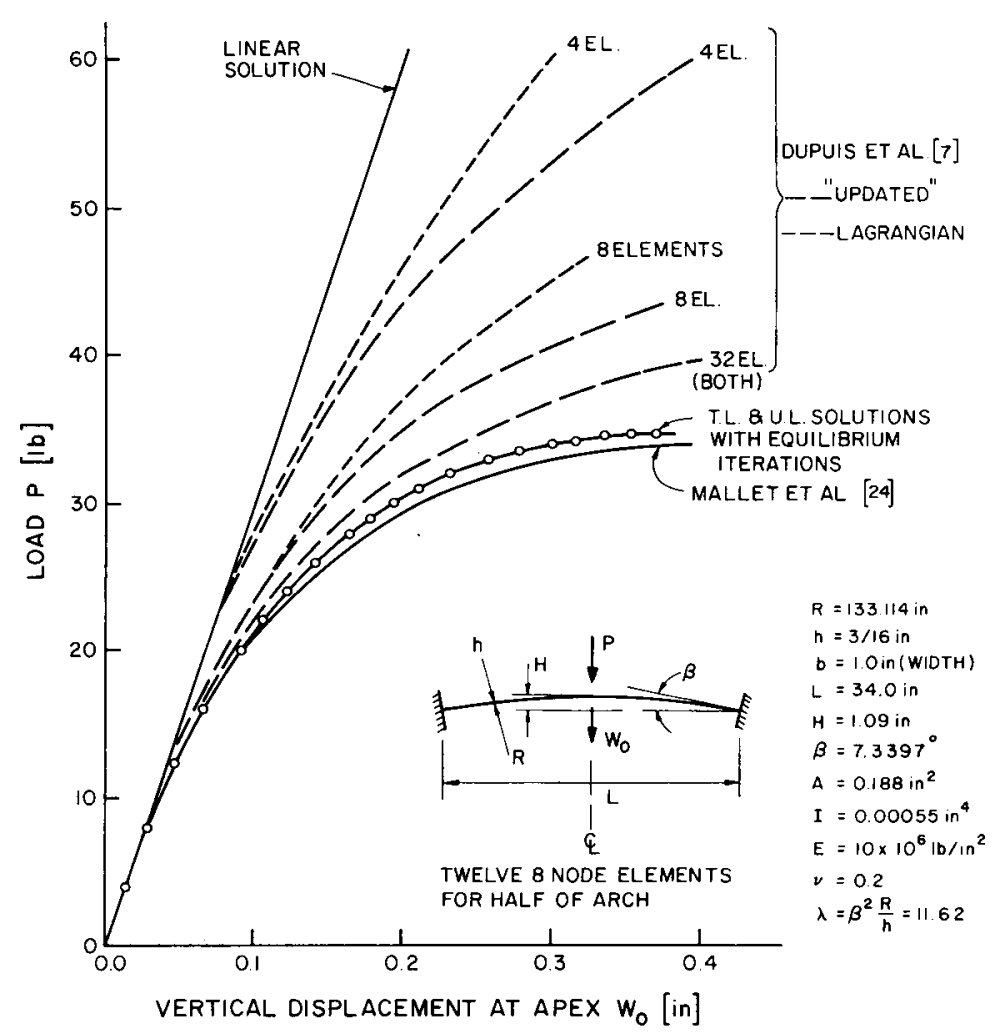

Figure 10. Load-deflection curve for a shallow arch under concentrated load 


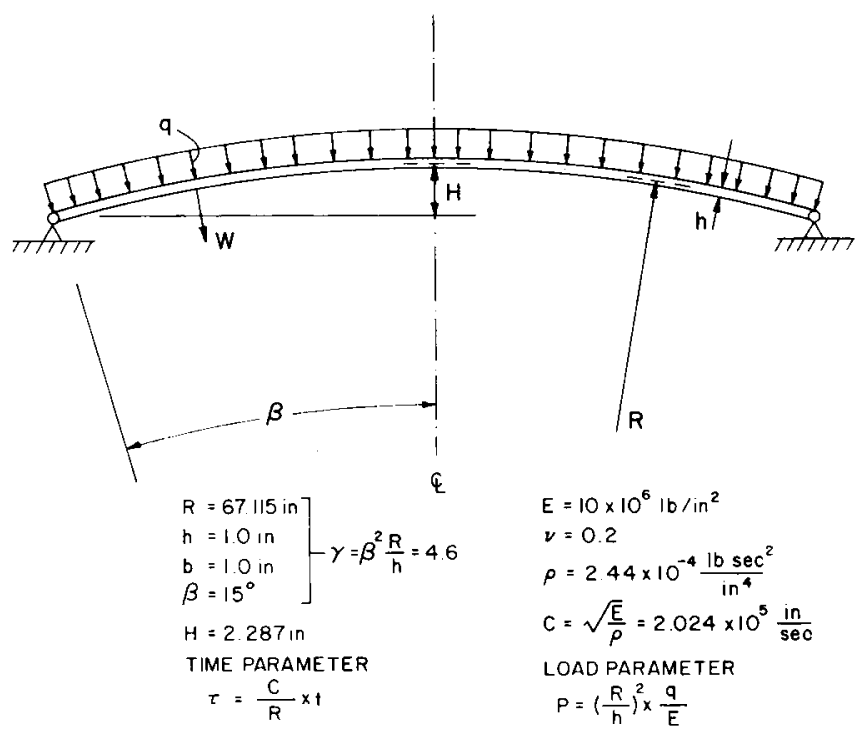

Figure 11. Simply supported shallow arch

7 psi. These constants are based on an analytical and experimental investigation of the rubber sheet by Iding. ${ }^{19}$ The finite element mesh used in the analysis is presented in Figure 13.

Figure 14 shows the static load deflection curves for different points on the sheet. Only five equal load increments with an average of four equilibrium iterations have been used to reach the final load position with a displacement of more than 11 in at point $B$. At this stage GreenLagrange strains of more than 4.5 are measured, see Figure 15. The results obtained are in

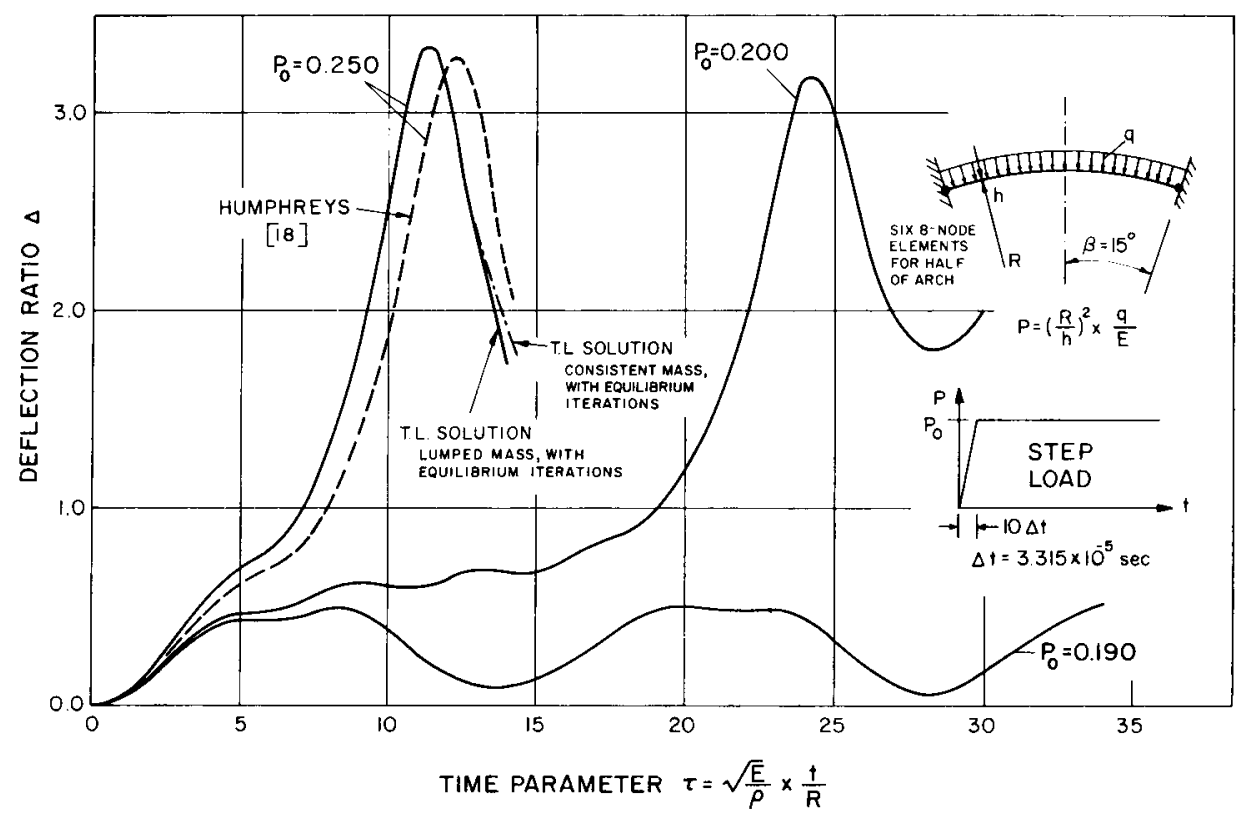

Figure 12. Dynamic snap-through of a shallow circular arch Wilson $\theta$ method, $\theta=1.4$ 

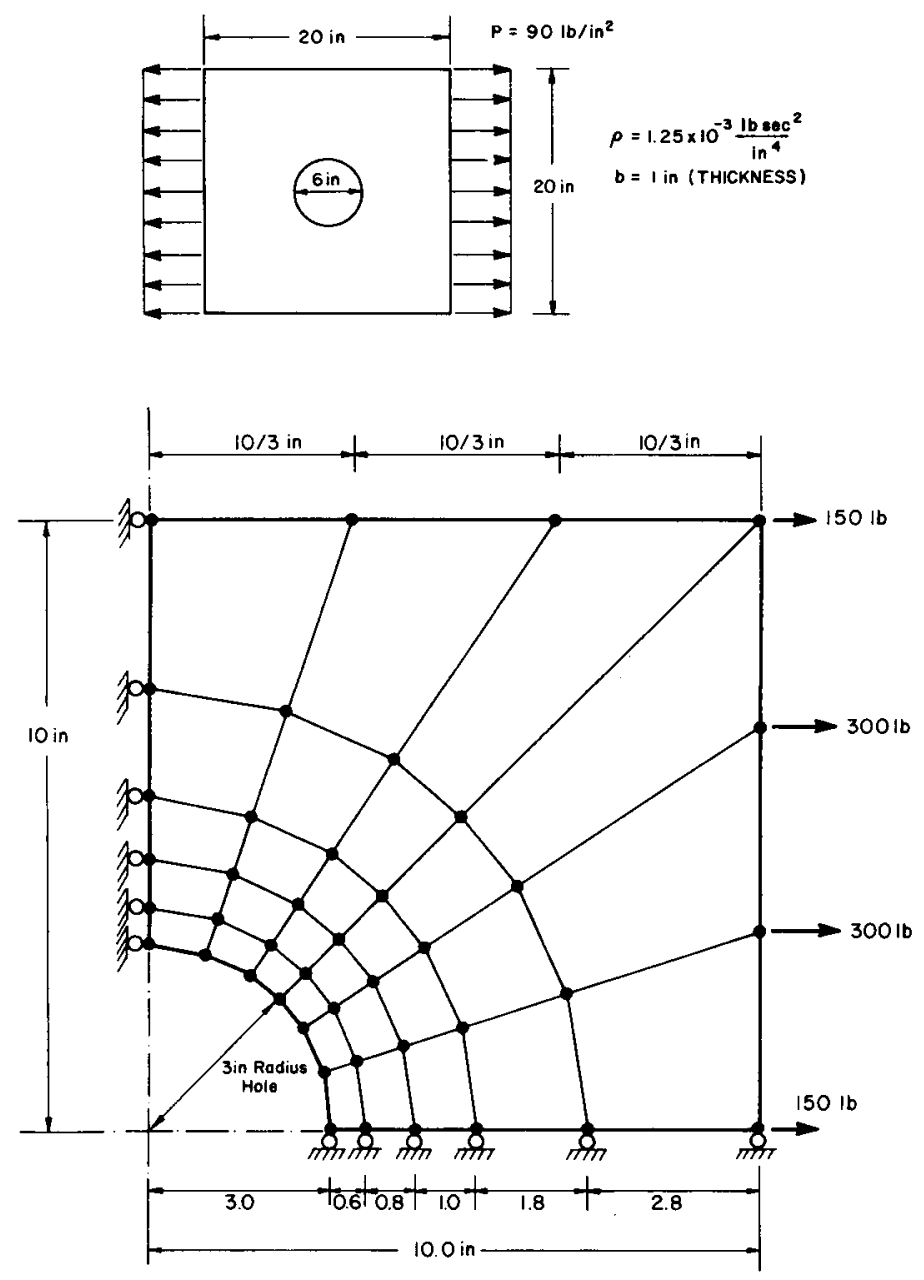

Figure 13. Finite element mesh of rubber sheet with hole

excellent agreement with those of Iding. The results of Iding have been obtained with the computer program developed in Reference 19, but are not given in the Reference.

The dynamic analysis was performed for the step load shown in Figure 16 using the Wilson $\theta$ and Newmark integration schemes with $\Delta t / T_{f} \cong 120$.

Figure 16 compares the displacement response predicted using the two integration methods. As is seen, practically the same response was calculated using the Wilson $\theta$ and the Newmark methods. In addition, it should be noted that identical solutions have been obtained using either integration scheme and an interval of stiffness reformation of 10, 5 or 1 time steps (see Table II). This should be expected, since the solution is unique for the selected time step $\Delta t$.

\section{Elastic-plastic large displacement dynamic analysis of a third spherical shell}

The dynamic response of the spherical shell in Figure 17 subjected to a distributed step pressure $p=600 \mathrm{lb} / \mathrm{in}^{2}$ was calculated. The material was assumed to obey the von Mises yield condition with linear isotropic hardening. The purpose of this analysis was to compare the results obtained 


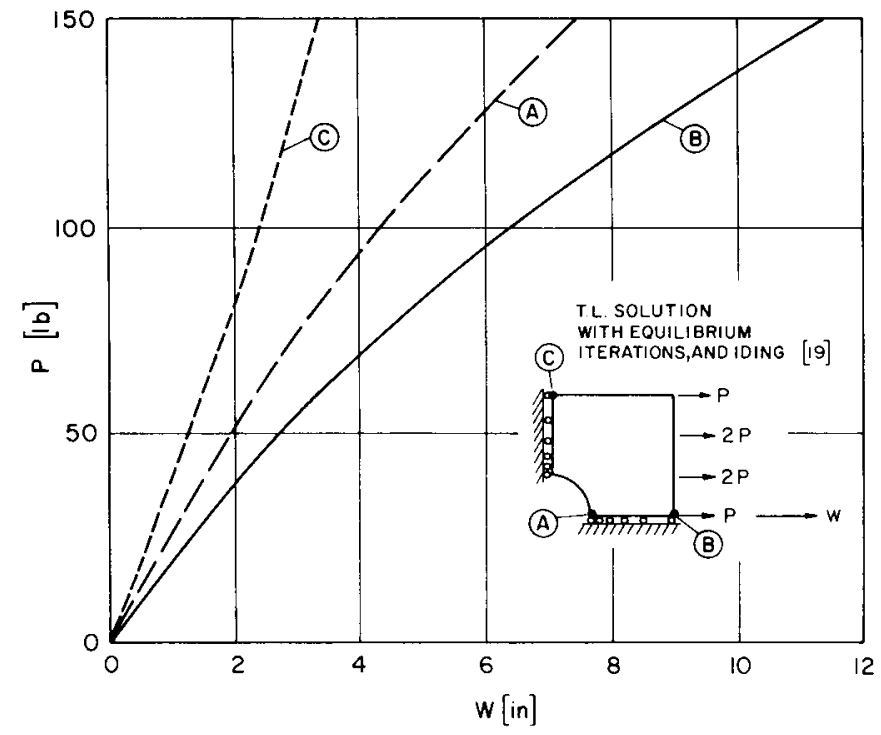

Figure 14. Static load-deflection curve for a rubber sheet with hole

using the various non-linear large displacement formulations available for elastic-plastic response calculations.

Figure 17 shows the dynamic response of the cap predicted using the Newmark time integration scheme in linear analysis, materially non-linear only analysis, i.e. assuming small displacements and small strains, and combined geometrically and materially non-linear analysis. In the fully non-linear analysis the solutions using the T.L., U.L.(T) and U.L.(J) formulations have been obtained. It is observed that all three formulations predict essentially the same response. The reason for obtaining almost identical solutions lies partly in that the mathematical representation of the yield function is almost the same in the 2nd Piola-Kirchhoff stress space and the Cauchy stress space. Namely, in problems of small strains but large rotations, such as in the

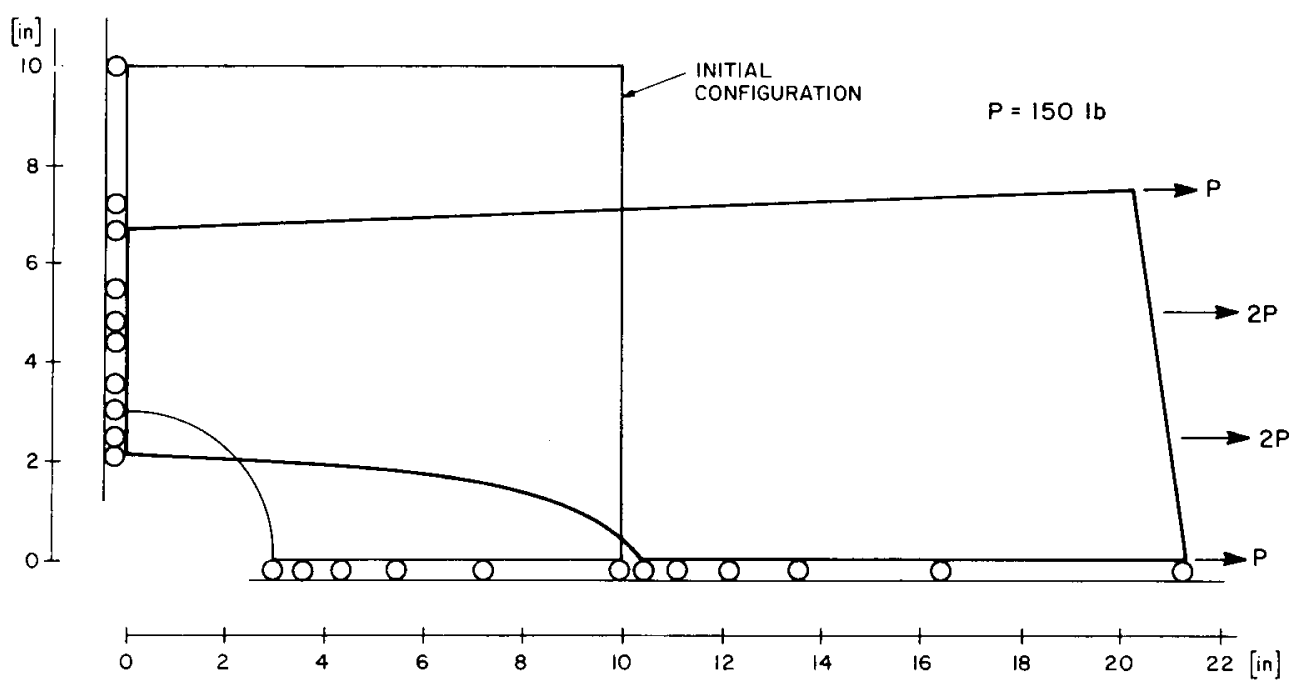

Figure 15. Deformed configuration drawn to scale of rubber sheet with hole (static analysis) 


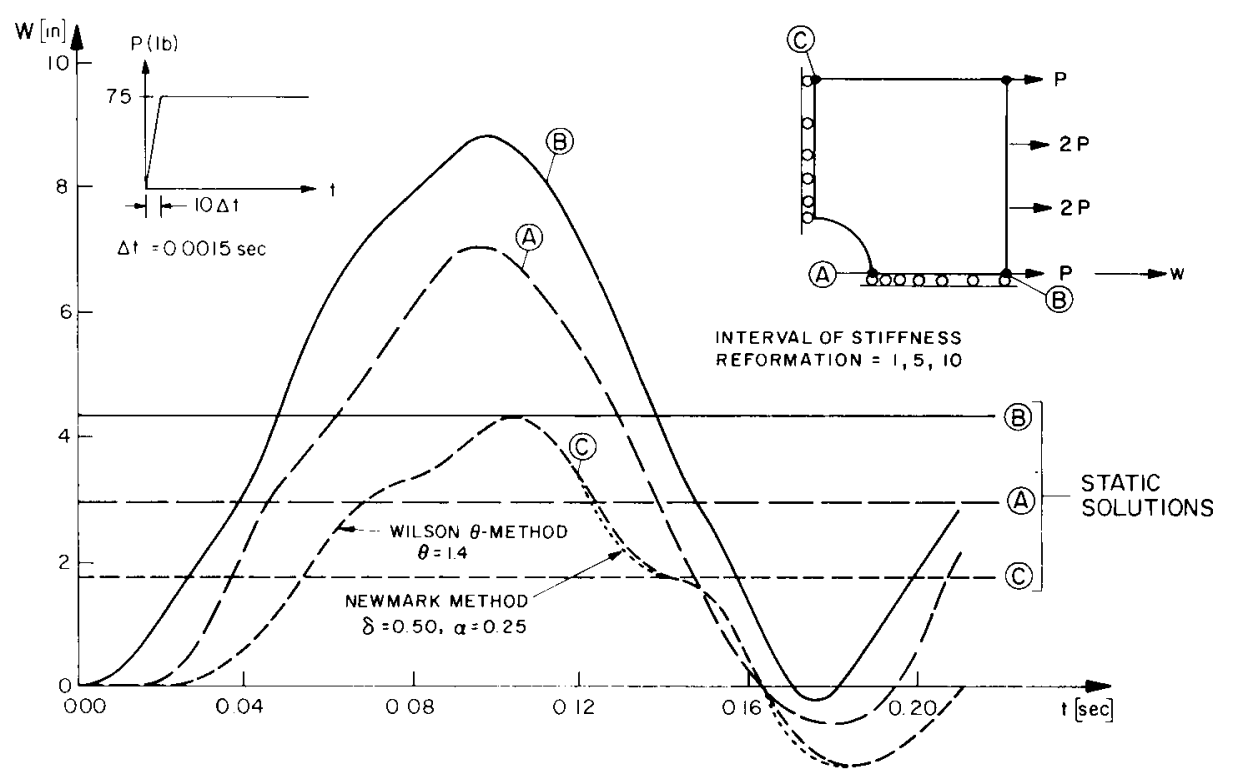

Figure 16. Displacements versus time for rubber sheet with hole, T.L. solution with equilibrium iterations

analysis of shells, the physical components of the Cauchy stress tensor in rotated (surface) co-ordinates are approximately equal to the Cartesian components of the 2 nd Piola-Kirchhoff stress tensor.

The solutions in Figure 17 demonstrate the effect of including different degrees of nonlinearities. It is observed that the materially non-linear only solution differs a great deal from the linear elastic response, and that the effect of large displacements is also significant. The decrease in amplitude of vibration and increase in the mean deflection of the shell when non-linearities are taken into account should be noted.

The response of the cap was also calculated using the Wilson $\theta$ method, which gave practically the same results. ${ }^{4}$

A comparison of the results obtained in this study with those calculated by Nagarajan ${ }^{29}$ is given in Figure 18. Nagarajan used degenerate isoparametric elements, in which it is assumed that the transverse normal stresses are negligibly small. This assumption affects the effective stress patterns which control plastic loading and contributes to the different response predicted in his study.

\section{CONCLUSIONS}

The objective in this paper was to review, derive and evaluate finite element formulations for general non-linear static and dynamic analysis which have been implemented in the search for the most effective procedure. ${ }^{3}$ The formulations have been derived from general principles of continuum mechanics and include material, large displacement and large strain non-linearities. The conceptual difference between the formulations is the reference configuration that is used for the linearization of the incremental equations of motion. In the T.L. formulation the initial configuration is used as reference, whereas in the U.L. formulations, the reference configuration corresponds to the last calculated configuration. 


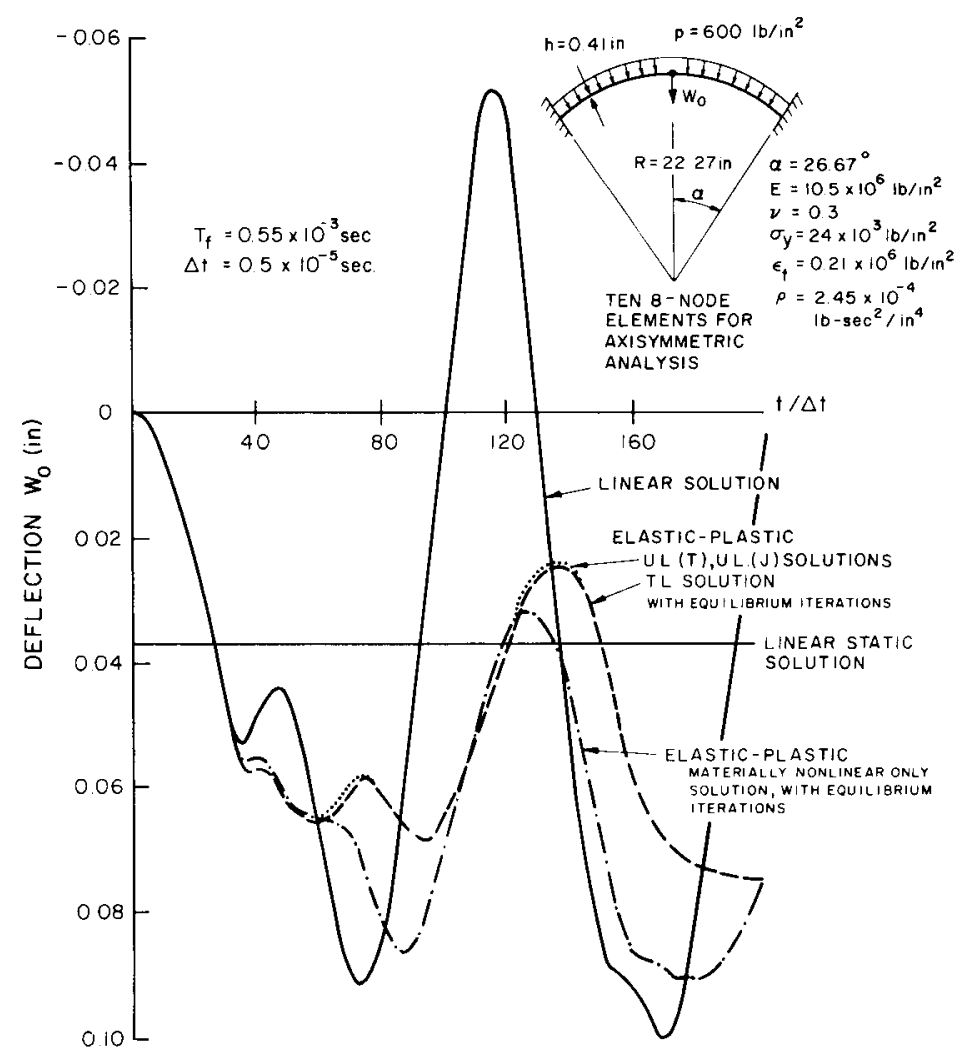

Figure 17. Large displacement dynamic elastic-plastic analysis of spherical cap. Newmark method. $\delta=0 \cdot 50, x=0 \cdot 25$

A first important observation is that provided the constitutive tensors are defined appropriately, all formulations give the same numerical results. The only advantage of using one formulation rather than the others is its better numerical effectiveness. Theory and sample analyses show that in small strain but large displacement analyses the differences which arise by using the same material constants in the formulations-because, for instance, a clear definition of the constants may not be available-can be expected to be small.

With regard to the numerical operations required, Table I shows that all matrices of the formulations have corresponding patterns of zero elements, except for the linear strain-displacement transformation matrices. In the T.L. formulation, this matrix is full, because of the initial displacement effect in the linear strain terms. Therefore, the calculation of the element matrices requires less time in the U.L. formulations.

An advantage of the T.L. formulation is that the derivatives of the interpolation functions are with respect to the initial configuration, and therefore need only be formed once, if they are stored on back-up storage for use in all load steps. However, in practice, the use of tape or disc to store and retrieve the required derivatives in each step may be more costly than simply to recalculate them, and, in particular, the required storage is a problem size governing factor since saturation of back-up storage may be reached. Auxiliary storage considerations are particularly important, if a considerable amount of stress and strain history need to be stored already.

It should be noted that the U.L. formulations are quite different from the moving co-ordinate formulation presented in the survey paper by Stricklin and many others, ${ }^{38}$ and the updated 


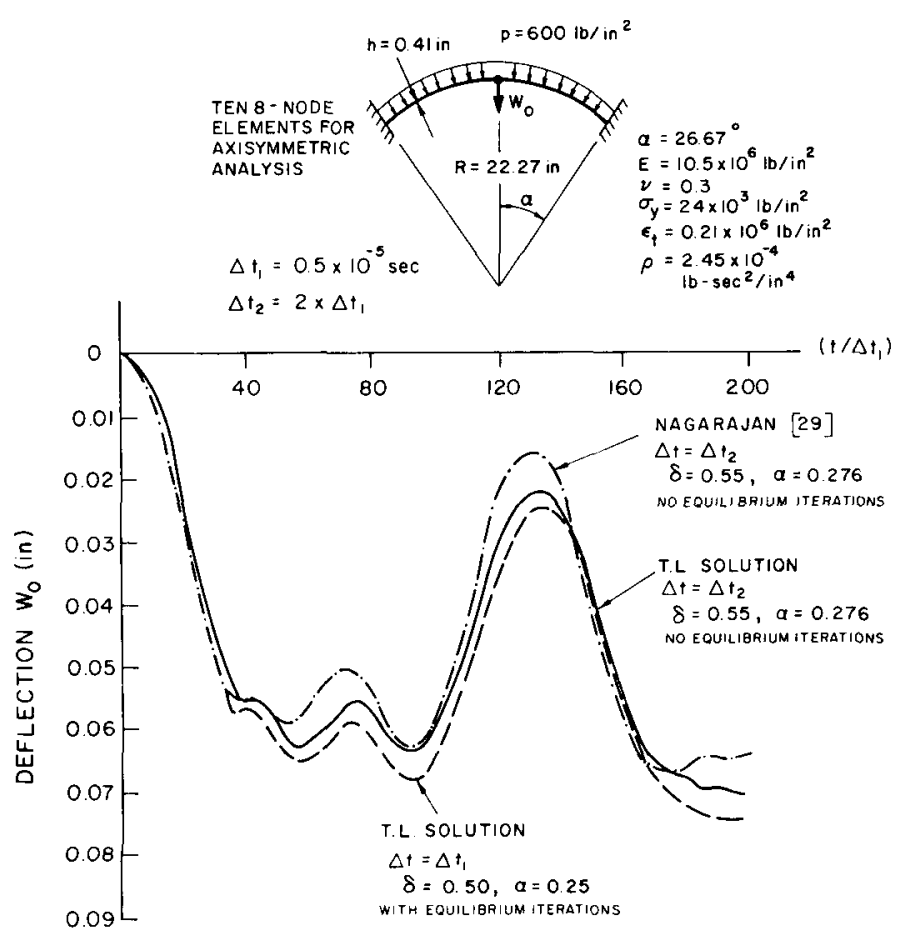

Figure 18. Large displacement dynamic elastic-plastic analysis of spherical cap, Newmark method

formulation, which was used in the comparative study by Dupuis and many others. ${ }^{7}$ The incremental moving co-ordinate formulation surveyed by Stricklin and many others, ${ }^{38,10,35}$ was stated to be restricted to small strains and have distinct computational disadvantages. These conclusions do not apply to the U.L. formulations used here. The 'updated' formulation employed by Dupuis and many others, in their comparative study of this formulation versus a Lagrangian formulation did not give satisfactory results. ${ }^{7}$ However, using the U.L. formulation with isoparametric elements as presented in this paper, the results are as good as those obtained using the T.L. formulation. The only errors are due to the numerical solution of the governing continuum mechanics equations.

In general, using both the T.L. and the U.L. formulations equilibrium iterations should be performed in order to ensure an accurate solution and possibly dispense with the calculation of a new non-linear stiffness matrix in each load step. If no equilibrium iterations are carried out, the linearization can introduce uncontrolled large errors. In the elastic and hyperelastic analyses presented here, it was possible to calculate the stresses in the configuration at time $t+\Delta t$ directly from the corresponding total strains. Therefore, the non-linear finite element equations have been solved 'exactly' within the assumptions of the time integration scheme and the convergence limit of the iteration. In path dependent problems this is not possible and total stresses are calculated by adding increments in stresses.

An important consideration in path dependent problems is the definition and calculation of the constitutive tensors, which depends on the stress and strain history. A great deal of additional research is still required to identify various materials. Using the T.L. formulation, the effort to implement a non-linear constitutive relation can, in some cases, be less than in the U.L. formulation; however, the appropriate material constants need be available. It is the ease of implementing 
a new material law in a general non-linear analysis program that makes the T.L. formulation very attractive.

In conclusion, both the U.L. or T.L. formulations can effectively be used in a general nonlinear analysis program. It depends largely on the program design and the material constants available which formulation is most effective.

\section{APPENDIX}

\section{Notation}

The following convention for tensor and vector subscripts and superscripts is employed:

A left superscript denotes the time of the configuration in which the quantity occurs.

A left subscript can have two different meanings. If the quantity considered is a derivative, the left subscript denotes the time of the configuration, in which the co-ordinate is measured with respect to which is differentiated. Otherwise the left subscript denotes the time of the configuration in which the quantity is measured.

Right lower case subscripts denote the components of a tensor or vector. Components are referred to a fixed Cartesian co-ordinate system; $i, j, \ldots=1,2,3$. Differentiation is denoted by a right lower case subscript following a comma, with the subscript indicating the co-ordinate with respect to which is differentiated.

$$
\begin{aligned}
& { }^{0} A,{ }^{t} A,{ }^{t+\Delta t} A=\text { Area of body in configuration at time } 0, t, t+\Delta t \\
& { }_{0}^{i} C_{i j r s},{ }_{t}^{i} C_{i j r s}=\text { Component of constitutive tensor at time } t \text { referred to configuration at } \\
& \text { time } 0, t \\
& { }_{0} C_{i j r s},{ }_{t} C_{i j r s}=\text { Component of tangent constitutive tensor at time } t \text { referred to configuration } \\
& \text { at time } 0, t \\
& { }^{t+\Delta t} f_{i}, t+\Delta t f_{t}=\text { Component of body force vector per unit mass in configuration at time } \\
& t+\Delta t \text { referred to configuration at time } 0, t+\Delta t \\
& h_{k}=\text { Finite element interpolation function associated with nodal point } k \\
& (i)=\text { Superscript indicating number of iteration } \\
& { }^{0} n_{i},{ }^{t} n_{i},{ }^{t+\Delta t} n_{i}=\text { Component of surface normal in configuration at time } 0, t, t+\Delta t \\
& { }^{t+\Delta t} p=\text { Pressure load at time } t+\Delta t \\
& { }^{t+\Delta} \mathscr{R}=\text { External virtual work expression corresponding to configuration at time } \\
& t+\Delta t \text {, defined in equation (8) } \\
& r, s=\text { Natural element co-ordinates } \\
& { }_{0}^{t} S_{i j},{ }_{0}^{t+\Delta t} S_{i j}=\text { Component of } 2 \text { nd Piola-Kirchhoff stress tensor in configuration at time } \\
& t, t+\Delta t \text { referred to configuration at time } 0 \\
& { }^{t+\Delta t} S_{i j}=\text { Component of } 2 \text { nd Piola-Kirchhoff stress tensor in configuration at time } \\
& t+\Delta t \text { referred to configuration at time } t \\
& { }_{0} S_{i j},{ }_{t} S_{i j}=\text { Component of 2nd Piola-Kirchhoff stress increment at time } t \\
& t, t+\Delta t=\text { time } t \text { and } t+\Delta t \text {, before and after time increment } \Delta t \\
& { }^{t+\Delta t} t_{i},{ }_{t}+\Delta t+\Delta t t_{i}=\text { Component of surface traction vector in configuration at time } t+\Delta t \text {, } \\
& \text { referred to configuration at time } 0, t+\Delta t \\
& { }^{t} u_{i},{ }^{t+\Delta t} u_{i}=\text { Component of displacement vector from initial position at time } 0 \text { to con- } \\
& \text { figuration at time } t, t+\Delta t \\
& u_{i}=\text { Increment in displacement component, } u_{i}={ }^{t+\Delta t} u_{i}-{ }^{t} u_{i} \\
& { }^{t} u_{i}^{k}=\text { Displacement component of nodal point } k \text { in configuration at time } t \\
& u_{i}^{k}=\text { Increment in }{ }^{t} u_{i}^{k}
\end{aligned}
$$




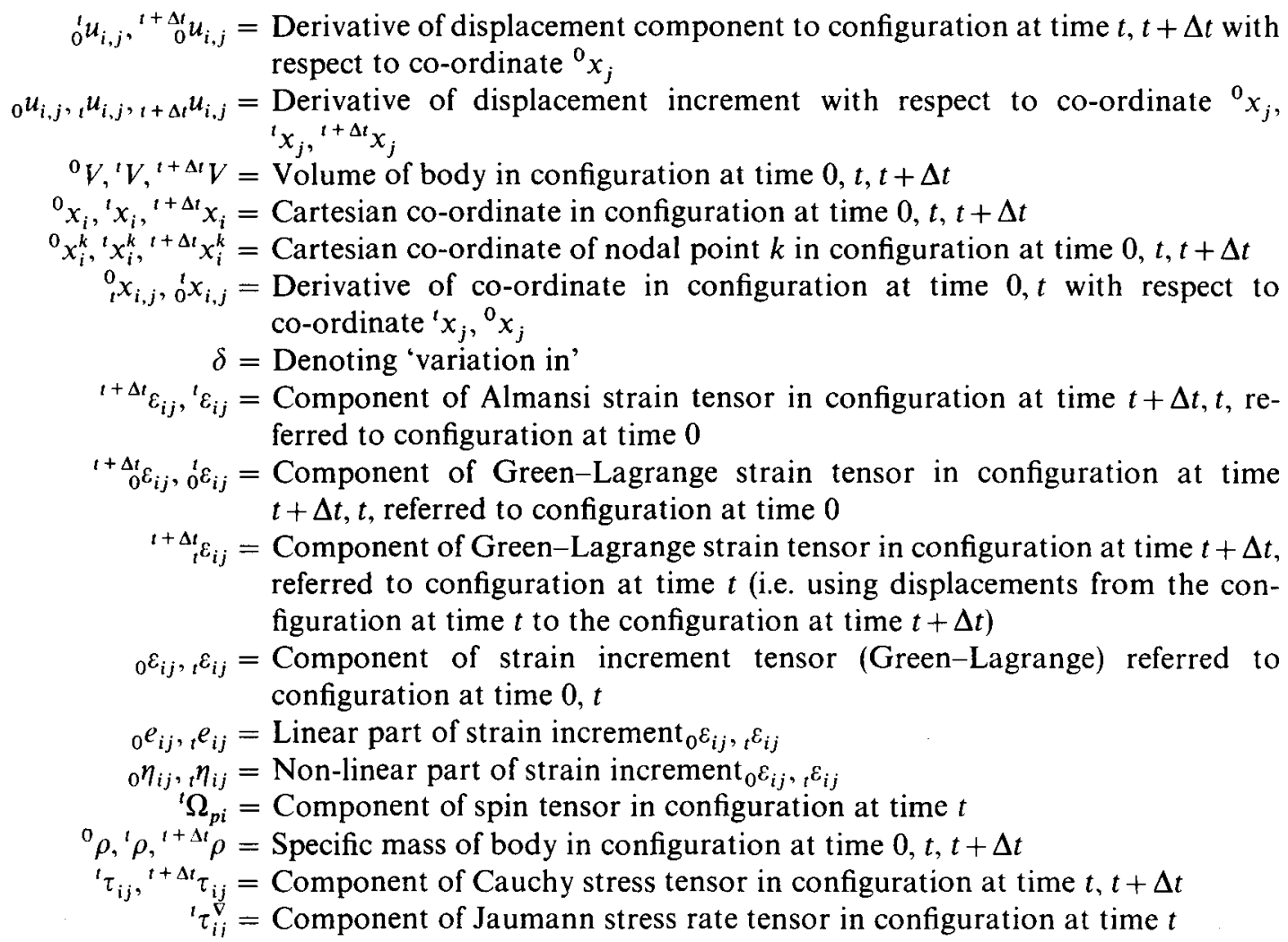

Matrices

${ }_{0}^{t} \mathbf{B}_{L},{ }_{t}^{t} \mathbf{B}_{L}=$ Linear strain-displacement matrix in configuration at time $t$ referred to configuration at time $0, t$

${ }_{0}{ }_{0} \mathbf{B}_{N L},{ }_{l}^{\prime} \mathbf{B}_{N L}=$ Non-linear strain-displacement matrix in configuration at time $t$ referred to configuration at time $0, t$

${ }_{0} \mathbf{C}, \mathbf{C}=$ Tangent material property matrix at time $t$ and referred to configuration at time $0, t$

${ }_{0}^{t} \mathbf{F},{ }_{t}^{t} \mathbf{F}=$ Vector of nodal point forces in configuration at time $t$ (referred to configuration at time $0, t$ )

${ }_{0}^{t} \mathbf{K}_{L},{ }_{t}^{t} \mathbf{K}_{L}=$ Linear strain stiffness matrix in configuration at time $t$ (referred to configuration at time $0, t)$

${ }_{0}^{t} \mathbf{K}_{N L},{ }_{t}^{t} \mathbf{K}_{N L}=$ Non-linear strain stiffness matrix in configuration at time $t$ (referred to configuration at time $0, t$ )

$\mathbf{M}=$ Mass matrix

${ }^{t+\Delta t} \mathbf{R}=$ Vector of external loads in configuration at time $t+\Delta t$

${ }_{0}^{t} \mathbf{S},{ }_{0}^{i} \hat{\mathbf{S}}=2$ nd Piola-Kirchhoff stress matrix and vector in configuration at time $t$ and referred to configuration at time 0

${ }^{\prime} \tau,{ }^{\prime} \hat{\tau}=$ Cauchy stress matrix and vector in configuration at time $t$

${ }^{t} \mathbf{u},{ }^{t+\Delta t} \mathbf{u}=$ Vector of displacements at time $t, t+\Delta t$

$\mathbf{u}=$ Vector of incremental displacements at time $t$ 


\section{REFERENCES}

1. J. H. Argyris, P. C. Dunne and T. Angelopoulos, 'Nonlinear oscillations using the finite element technique', Computer Meth. Appl. Mech. Engng, 2, 203-250 (1973).

2. K. J. Bathe and E. L. Wilson, "NONSAP-A general finite element program for nonlinear dynamic analysis of complex structures', Paper M3/1, Proc. 2nd Int. Conf. Struct. Mech. Reactor Technology, Berlin (1973).

3. K. J. Bathe, E. L. Wilson and R. H. Iding, "NONSAP-A structural analysis program for static and dynamic response of nonlinear systems', SESM Report No. 74-3, Dept. of Civ. Engng, Univ. of California, Berkeley (1974).

4. K. J. Bathe, H. Ozdemir and E. L. Wilson, 'Static and dynamic geometric and material nonlinear analysis', SESM Report No. 74-4, Dept. Civ. Engng, Univ. of California, Berkeley (1974).

5. K. J. Bathe and E. L. Wilson, 'Stability and accuracy analysis of direct integration methods', Int. J. Earthq. Engng Struct. Dyn., 1, 283-29l (1973).

6. T. Belytschko and B. J. Hsieh, 'Nonlinear transient analysis of shells and solids of revolution by convected elements', AIAA paper No. 73-359, AIAA/ASME/SAE 14th Structures, Struct. Dyn. Materials Conf., Williamsburg, Virginia (1973).

7. G. A. Dupuis, H. D. Hibbitt, S. F. McNamara and P. V. Marcal, 'Nonlinear material and geometric behavior of shell structures', Computers Struct., 1, 223-239 (1971).

8. I. Farhoomand, 'Nonlinear dynamic stress analysis of two-dimensional solids', Ph.D. Dissertation, Univ. of California, Berkeley, 1970.

9. C. A. Felippa, 'Refined finite element analysis of linear and nonlinear two-dimensional structures', SESM Report No. 66-22, Dept. Civ. Engng, Univ. of California, Berkeley (1966).

10. C. A. Felippa and P. Sharifi, 'Computer implementation of nonlinear finite element analysis', Proc. Symp. ASME, Detroit (1973).

11. Y. C. Fung, Foundations of Solid Mechanics, Prentice-Hall, Englewood Cliffs, N.J., 1965.

12. A. Gjelsvik and S. R. Bodner, 'The energy criterion and snap buckling of arches', J. Engng Mech. Div., ASCE, 88, 87-134 (1962).

13. E. Haug and G. H. Powell, 'Finite element analysis of nonlinear membrane structures', SESM Report No. 72-7, Dept. of Civ. Engng, Univ. of California, Berkeley (1972).

14. B. J. Hartz and N. D. Nathar, 'Finite element formulation of geometrically nonlinear problems of elasticity', Recent Advances in Matrix Methods of Structural Analysis and Design, 1st Japan-U.S. Seminar Matrix Meth. Struct. Analysis and Design, Univ. of Alabama Press, 415-440 (1971).

15. J. H. Heifitz and C. J. Costantino, 'Dynamic response of nonlinear media at large strains', J. Engng Mech. Div., ASCE, 98, 1511-1527 (1972).

16. H. D. Hibbitt, P. V. Marcal and J. R. Rice, 'Finite element formulation for problems of large strain and large displacements', Int. J. Solids Struct. 6, 1069-1086(1970).

17. J. T. Holden, 'On the finite deflections of thin beams', Int. J. Solids Struct. 8, 1051-1055 (1972).

18. J. S. Humphreys, 'On dynamic snap buckling of shallow arches', AIAA J. 4, 878-886 (1966).

19. R. H. Iding, 'Identification of nonlinear materials by finite element methods', SESM Report No. 73-4, Dept. of Civ. Engng, Univ. of California, Berkeley (1973)

20. P. S. Jensen, 'Transient analysis of structures by stiffly stable methods', Computers Struct., 4, 615-626 (1974).

21. H. S. Kornishin and F. S. Isanbaeva, Flexible Plates and Panels, (in Russian), Nauka, Moscow, 1968.

22. P. K. Larsen, 'Large displacement analysis of shells of revolution, including creep, plasticity and viscoelasticity', SESM Report No. 71-22, Dept. of Civ. Engng, Univ. of California, Berkeley (1971).

23. E. H. Lee, 'Elastic-plastic deformation at finite strains', J. Appl. Mech., Trans. ASME, 38 (1969).

24. R. H. Mallet and L. Berke, 'Automated method for the large deflection and instability analysis of 3-dimensional truss and frame assemblies', AFFDL-TR-66-102 (1966).

25. L. E. Malvern, Introduction to the Mechanics of a Continuum Medium, Prentice-Hall, Englewood Cliffs, N.J., 1969.

26. P. V. Marcal, 'The effect of initial displacements on problems of large deflection and stability', Tech. Report ARPA E54, Brown University, Division of Engineering (1967).

27. J. F. Mescall, 'Large deflection of spherical shells under concentrated loads', J. Appl. Mech. 32, 936-938 (1965).

28. D. W. Murray and E. L. Wilson, 'Finite element large deflection analysis of plates', J. Engng Mech. Div., ASCE, 94, 143-165 (1965).

29. S. Nagarajan, 'Nonlinear static and dynamic analysis of shells of revolution under axisymmetric loading', $S E S M$ Report No. 73-11, Dept. of Civ. Engng, Univ. of California, Berkeley (1973).

30. J. F. McNamara, 'Incremental stiffness method for finite element analysis of the nonlinear dynamic problem', Ph.D. Thesis, Dept. of Civ. Engng, Brown University, 1972.

31. R. E. Nickell, 'Direct integration methods in structural dynamics', J. Engng Mech. Div., ASCE, 99, 303-317 (1973).

32. J. T. Oden, 'Finite element applications in nonlinear structural analysis', Proc. Symp. Application Finite Element Meth. Civ'. Engng, Nashville, Tenn. (1969).

33. J. T. Oden, Finite Elements of Nonlinear Continua, McGraw-Hill, New York, 1972.

34. H. Schreyer and E. Masur, 'Buckling of shallow arches', J. Engng Mech. Div., ASCE, 92, 1-19 (1966).

35. P. Sharifi and E. P. Popov, 'Nonlinear buckling analysis of sandwich arches', J. Engng Mech. Div., ASCE, 97, $1397-1412$ (1970). 
36. P. Sharifi and D. N. Yates, 'Nonlinear thermo-elastic-plastic and creep analysis by the finite element method', AIA A Paper No. 73-358, AIAA/ASME/SAE 14th Structures, Struct. Dyn. Materials Conf., Williamsburg, Virginia (1973).

37. J. A. Stricklin, 'Geometrically nonlinear static and dynamic analysis of shells of revolution', High Speed Computing Elastic Struct., Proc. Symp. IUTAM, Univ. of Liege, 383-411.

38. J. A. Stricklin, W. A. Von Riesemann, J. R. Tillerson and W. E. Haisler, 'Static geometric and material nonlinear analysis', Advances in Computational Methods in Computational Methods in Structural Mechanics and Design, 2nd U.S.-Japan Seminar Matrix Meth. Struct. Analysis and Design, Univ. of Alabama Press, $301-324$ (1972).

39. S. Yaghmai, 'Incremental analysis of large deformations in mechanics of solids with applications to axisymmetric shells of revolution', SESM Report No. 69-17, Dept. of Civ. Engng, Univ. of California, Berkeley (1968).

40. S. Yaghmai and E. P. Popov, 'Incremental analysis of large deflections of shells of revolution', Int. J. Solids Struct. 7, 1375-1393 (1971).

41. Y. Yamada, 'Incremental formulation for problems with geometric and material nonlinearities', Advances in Computational Methods in Structural Mechanics and Design, 2nd U.S.-Japan Seminar Matrix Meth. Struct. Analysis Design, Univ. of Alabama Press, 325-355 (1972).

42. C. H. Yeh, 'Large deflection dynamic analysis of thin shells using the finite element method', SESM Report No. 70-78, Dept. of Civ. Engng, Univ. of California (1970).

43. O. C. Zienkiewicz, The Finite Element Method in Engineering Science, McGraw-Hill, London, 1971. 